\title{
COLORADOSCHOOLOFMINES
}

EARTH•ENERGY•ENVIRONMENT

Division OF ECONOMICS AND BUSINESS

WORKING PAPER SERIES

\section{Energy efficiency and emissions intensity standards}

\author{
Harrison Fell \\ Daniel Kaffine \\ Daniel Steinberg
}

\author{
Working Paper 2015-09 \\ http: //econbus.mines . edu/working-papers/wp201509.pdf \\ Colorado School of Mines \\ Division of Economics and Business \\ 1500 Illinois Street \\ Golden, CO 80401
}

November 2015

(c) 2015 by the listed authors. All rights reserved. 
Colorado School of Mines

Division of Economics and Business

Working Paper No. 2015-09

November 2015

Title:

Energy efficiency

and emissions intensity standards*

Author(s):

Harrison Fell

Division of Economics and Business

Colorado School of Mines

Golden, CO 80401-1887

hfell@mines.edu

Daniel Kaffine

Department of Economics

University of Colorado, at Boulder

daniel.kaffine@colorado.edu

Daniel Steinberg

National Renewable Energy Lab

daniel.steinberg@nrel.gov

\begin{abstract}
In order to comply with the Environmental Protection Agency's (EPA) recently released regulations governing greenhouse gas emissions from power plants, states are given the option to implement rate-based emissions intensity standards for the power sector. One well-known consequence of rate-based emissions standards is that in addition to encouraging substitution towards less emissions-intensive sources, they also subsidize output, and thus are considered by economists to be inferior to a first-best solution. However, the exiting literature has not considered energy efficiency decisions within the framework of intensity standards. This omission is particularly problematic in the context of the power sector, where energy efficiency has been considered an important channel of cost-minimizing emissions reductions. To encourage end-use efficiency measures under an intensity standard EPA allows states to credit electricity savings as a means of complying with the rule by treating them as a form of zero-emissions output. In this paper we investigate the role of energy efficiency choices in rate-based emissions intensity standards. We show that when demand for energy services is perfectly inelastic, crediting efficiency measures can recover the first-best allocation. This approach extends the output subsidy in a traditional intensity standard to energy efficiency, thereby eliminating the distortion that favors electricity generation over energy efficiency. However, when demand for energy services exhibits some elasticity, crediting energy efficiency can no longer recover first-best. While crediting energy efficiency removes the relative distortion between energy generation and energy efficiency, it distorts the equilibrium level of both energy generation and energy efficiency via an energy services subsidy. Simulations calibrated to the electricity sector in Texas examine the above issues numerically, as well as explore the implications of alternative energy efficiency crediting schemes.
\end{abstract}

${ }^{*}$ We thank participants at the 3rd Annual Front Range Energy Camp and 2015 AERE Summer Conference for comments. Ed Balistreri, Jared Carbone, Jonathan Hughes, Derek Lemoine, and Andrew Yates provided useful comments. 


\section{Introduction}

Emissions intensity standards have recently emerged as a policy tool of interest for curbing greenhouse gas emissions. ${ }^{1} \quad$ For example, California instituted a Low Carbon Fuel Standard (LCFS) for transportation fuels which sets declining limits for the average emissions per gallon of fuel consumed (Holland, Hughes, and Knittel 2009). Similarly, under the Environmental Protection Agency's (EPA) recently released Clean Power Plan (CPP), which sets rate-based emissions targets (in lbs of CO2 per megawatt-hour) for the electric sector in every state, a Carbon Intensity Standard (CIS) is identified as an option for achieving compliance with the rate-based targets (Palmer and Paul 2015).

A well-known consequence of emission intensity standards is that they encourage substitution towards less emissions-intensive sources but also subsidize energy output, and thus are considered by economists to be inferior to the first-best solution of a Pigovian tax or equivalent cap and trade system. However, this output subsidy effect, and intensity standards more generally, have been considered in frameworks that do not explicitly incorporate energy efficiency choices. This omission is particularly noteworthy in the context of electricity, where energy efficiency has been considered an important channel of cost-minimizing emissions reductions. Indeed, to encourage energy efficiency measures for the electricity sector, crediting of energy efficiency as a means of complying with the intensity standard is identified as an option under the EPA's proposed rule. In this paper, we ask: Can emissions intensity standards recover the first-best solution when energy efficiency choices are

\footnotetext{
${ }^{1}$ Emissions intensity standards can be characterized simply as requiring the sum of emissions from all sources (the numerator) divided by total output (the denominator) to be less than some specified intensity target. Such standards are often referred to as "rate-based" policies, as opposed to "mass-based" policies that target total emission levels.
} 
considered, and under what conditions? How should regulators credit energy efficiency in an emissions intensity standard framework? What are the consequences of alternative crediting levels for the electricity sector?

The result that intensity standards cannot achieve a first-best outcome due to the implicit output subsidy has been well-established in the prior literature (Helfand 1991; Fullerton and Heutel 2010). ${ }^{2} \quad$ For example, in the context of the LCFS, Holland, Hughes, and Knittel (2009) show that the standard subsidizes below-average (but still emissions-creating) fuel sources, such that overall emissions may perversely increase. Recognizing this source of inefficiency, prior studies have advocated the coupling of intensity standards with additional instruments such as a fuel tax (Holland, Hughes, and Knittel 2009) or consumption tax (Holland 2012), or optimally adjusting the emissions ratings of each source (Lemoine 2013). While such approaches recover first-best, they also require the implementation of an additional instrument or complex manipulation of emission ratings. Furthermore, as energy efficiency measures are not considered in the above studies, it is unclear how decisions about energy efficiency are impacted by such standards. If some form of crediting energy efficiency within an intensity standard can recover first-best outcomes, such an approach may be more appealing relative to the multiple-policy instrument options prescribed in previous works.

To examine the role of energy efficiency in intensity standards policies, with a particular application to electricity markets, we develop a parsimonious model of the electricity sector, where households meet their demand for energy services by purchasing electricity or

${ }^{2}$ If there are additional market failures above and beyond the emissions externality, an intensity standard is not necessarily inferior to an emissions tax. For example, Holland (2012) finds that an intensity standard may dominate an emissions tax in the presence of leakage to unregulated regions. 
energy efficiency from firms. Firms decide which types of electricity generators, each with differing emissions intensities, they will dispatch, as well as how much energy efficiency to produce. ${ }^{3}$ We then consider the outcome of various forms of regulations regarding emissions and generation mixes.

We show that if demand for energy services is perfectly inelastic, then the traditional intensity standard is equivalent to an emissions tax coupled with a tax on energy efficiency. Relative to the first-best policy of an emission tax, due to the output subsidy effect there is too little investment in energy efficiency, and, similar to previous works on intensity standards, too much generation from low emission sources (e.g. natural gas-fired generation). However, crediting energy efficiency as a form of zero-emission generation (adding it to the denominator of the standard), can recover the first-best outcome. The intuition is that by crediting energy efficiency, the relative output subsidy distortion between generation and energy efficiency is eliminated. Importantly, this adjustment to the traditional intensity standard does not require the use of an additional instrument or optimal adjustments to emissions ratings to achieve first-best. However, when demand for energy services exhibits some elasticity, the intensity standard with energy efficiency credited as zero-emission generation can no longer recover first-best, though it may still be more efficient than the traditional intensity standard offering no efficiency crediting. This occurs because the intensity standard with crediting replaces the output subsidy of the traditional intensity standard with an energy services subsidy, leading to excessive consumption of energy services (both generation

\footnotetext{
${ }^{3}$ As our model is static, we abstract from the dynamic considerations associated with the fact that energy efficiency in practice is a characteristic bundled within a durable good (e.g. an Energy Star refrigerator). Instead, households are assumed to be able to purchase an amount of energy efficiency at some cost.
} 
and energy efficiency) relative to first-best. As such, a tradeoff emerges between removing the relative distortion between generation and energy efficiency, and creating a distortion in the absolute level of energy services consumed. Thus, the wedge between first-best and the intensity standard with crediting hinges on the degree of elasticity in the demand for energy services by households.

Finally, in a detailed numerical application calibrated to Electricity Reliability Corporation of Texas (ERCOT) region, we consider the relative efficiency of intensity standards versus emission tax policies under a range of energy efficiency crediting levels and under a variety of demand, market, and policy conditions. This simulation exercise highlights that the relatively simple theory model does well in predicting the general outcomes of a much more complicated electricity dispatch model with energy efficiency. The simulations also provide numerically relevant efficiency impacts of energy efficiency crediting in a wide range of settings, thereby providing guidance to policy makers in setting appropriate energy efficiency crediting rates.

Our paper provides several contributions. First, it incorporates energy efficiency choices into the theoretical literature on intensity standards, providing a new interpretation of intensity standards as an implicit tax on energy efficiency choices. Second, we show that in some cases, crediting energy efficiency can undo the adverse output subsidy effect created by intensity standards. Third, by incorporating energy efficiency in the simulation exercise in a theoretically-consistent manner, we provide a new framework for considering energy efficiency that goes beyond the current practice of ad hoc shifts in the demand or supply curve for electricity. Finally, our results provide policymakers guidance in terms of designing policies, particularly with respect to the proposed Clean Power Plan and the tradeoffs 
associated with crediting energy efficiency.

\section{Intensity standards and energy efficiency}

Our theoretical model examines the role of energy efficiency in emissions intensity standards for two different cases. In the first case, demand for energy services is assumed to be inelastic. We then examine intensity standards as compared to the first-best emissions tax under alternative crediting schemes for energy efficiency. In the second case, demand for energy services is assumed to exhibit some elasticity, and we again examine outcomes under intensity standards compared to the first-best emissions tax. While our exposition will focus on the electricity sector, the basic insights are relevant for other energy sectors where energy efficiency is a relevant choice (e.g. fuel).

Before analyzing the particular cases, we first describe the common components of the theoretical exercise. We begin by assuming the representative households' preferences do not include energy consumption or energy efficiency directly, but rather households have preferences for energy services such as heating, lighting, recreation, refrigeration, etc. These energy services are produced from both energy consumption and energy efficiency as follows:

$$
E S=E S(Q, \theta)
$$

where $E S(Q, \theta)$ is increasing in both arguments. The basic intuition is that energy services can either be provided by $Q$ electricity consumption or $\theta$ investments in energy efficiency. ${ }^{4}$

\footnotetext{
${ }^{4}$ For example, in the case of lighting, a household could receive the same level of energy services from a cheap 60 watt bulb as they could from "investing" in a more expensive energy-efficient LED bulb that uses the equivalent of 9 watts.
} 
While we assume consumers are indifferent to the source of production for $Q$, the general formulation in equation 1 allows for energy consumption and energy efficiency to have varying degrees of productivity and substitutability in terms of generating energy services.

On the production side, we assume a representative firm produces both electricity $Q$ and energy efficiency $\theta \cdot{ }^{5} \quad$ Total electricity production $Q(\mathrm{MWh})$ is produced from $N$ different generation technologies, each indexed by $i$, with quantities of $Q_{i}$ and emission generation rates of $\gamma_{i} \geq 0$ (tons per $\mathrm{MWh}$ ), such that: ${ }^{6}$

$$
Q=\sum_{i}^{N} Q_{i} .
$$

The marginal cost of producing the $q$ th unit of electricity for each technology is given as $c_{i}\left(q_{i}\right)$ and is assumed to be weakly increasing in $q_{i}$. The cost of producing energy efficiency is given by $e(\theta)$ and is assumed to be increasing and convex.

Emissions are assumed to generate constant marginal damages, and as such, the first-best policy response is to set a $\operatorname{tax} \tau$ equal to that marginal damage (e.g. $\$ 39$ dollars per ton of $\mathrm{CO}_{2}$ ). The alternative policy of the emissions intensity standard is modeled as:

$$
I \geq \frac{\sum_{i}^{N} \gamma_{i} Q_{i}}{f(\theta)+Q}
$$

\footnotetext{
${ }^{5}$ Our treatment collapses the complicated electricity sector into a tractable setup that nonetheless captures the key decisions regarding efficiency. As a consequence, it abstracts from other important issues such as deregulated versus regulated markets (Fabrizio, Rose, and Wolfram 2007), or behaviorial responses by consumers to electricity and energy efficiency prices (Allcott and Greenstone 2012; Ito 2014).

${ }^{6}$ The different technologies can be thought of as different fuel types such as coal or gas or wind, or even different technologies within a fuel type such as combined-cycle or combustion turbines for gas. We assume that $\gamma_{i}>0$ for at least some $i$, and that $\gamma_{i}>\gamma_{j}$ for some $i \neq j$.
} 
where $I>0$ is the intensity standard target (emissions per unit output) and $f(\theta)$ describes how energy efficiency is credited for the purposes of the intensity standard. For example, if energy efficiency is not credited (the traditional intensity standard), then $f(\theta)=0$, and if energy efficiency is credited one-for-one, then $f(\theta)=\theta$.

\subsection{Inelastic demand for energy services}

We first consider the case where demand for energy services is assumed to be inelastic, such that $\overline{E S}=E S(Q, \theta)$. With inelastic demand, consumer surplus is undefined, and as such we focus on the firm's problem of meeting the fixed energy service demand $\overline{E S}$ at minimum cost. As a reference point, consider the laissez-faire solution. In the absence of regulation, the firm's min-cost problem is to solve:

$$
\begin{gathered}
\min _{Q_{i}, \theta} \sum_{i}^{N} \int_{0}^{Q_{i}} c_{i}\left(q_{i}\right) d q_{i}+e(\theta) \\
\text { s.t. } \overline{E S}=\operatorname{ES}(Q, \theta) .
\end{gathered}
$$

Forming the Lagrangian and taking first order conditions yields the intuitive relationship:

$$
\frac{c_{i}\left(Q_{i}\right)}{\partial E S / \partial Q}=\frac{c_{j}\left(Q_{j}\right)}{\partial E S / \partial Q}=\frac{d e / d \theta}{\partial E S / \partial \theta} \quad \forall i, j
$$

whereby the firm equates the marginal cost of producing energy services across all generation technologies and energy efficiency. ${ }^{7}$ However, because emissions are not priced, the resulting

7 The denominator of equation 5 accounts for the transformation of generation or energy efficiency into energy services. An intuitive case to consider that aids exposition is where electricity and energy efficiency are perfect substitutes, such that $\partial E S / \partial Q=\partial E S / \partial \theta=1$. 
allocation is not first-best due to the standard externality problem.

\subsubsection{First-best emissions tax}

When the regulator sets an emissions $\operatorname{tax} \tau$ equal to the marginal external damage of emissions, the firm's Lagrangian is given by:

$$
L=\sum_{i}^{N} \int_{0}^{Q_{i}} c_{i}\left(q_{i}\right) d q_{i}+e(\theta)+\tau\left(\sum_{i}^{N} \gamma_{i} Q_{i}\right)+\lambda(\overline{E S}-E S(Q, \theta))
$$

where $\lambda$ is the shadow cost of the fixed energy service constraint. Relative to the case without regulation, the firm now must account for the additional cost associated with the tax on emissions. This leads to the following lemma, which will be useful when we examine the case of emissions intensity standards below.

Lemma 1. Under inelastic demand, the first-best allocation equates marginal costs of energy services net of the emissions externality. More energy efficiency is produced and generation is reduced, relative to the unregulated case.

Proof. All proofs are provided in the Appendix.

From the first-order conditions of this problem, we see that the first-best solution satisfies:

$$
\frac{c_{i}\left(Q_{i}\right)+\tau \gamma_{i}}{\partial E S / \partial Q}=\frac{c_{j}\left(Q_{j}\right)+\tau \gamma_{j}}{\partial E S / \partial Q}=\frac{d e / d \theta}{\partial E S / \partial \theta} \quad \forall i, j
$$

such that the marginal cost of energy services from each generation technology inclusive of emissions damages is equated across sources and with energy efficiency. Relative to the In that case, marginal costs are simply equated across all generators and energy efficiency. 
laissez-faire solution in equation 5 , the tax shifts generation from high-emission sources to lower emission sources, as well as provides an incentive for increased energy efficiency. ${ }^{8}$

\subsubsection{Emissions intensity standard with and without crediting}

We next consider whether or not an emissions intensity standard can recover the above condition for first-best regulation of the emissions externality. Given equation 3 as binding, the firm's Lagrangian problem is:

$$
L=\sum_{i}^{N} \int_{0}^{Q_{i}} c_{i}\left(q_{i}\right) d q_{i}+e(\theta)+\mu\left(\sum_{i}^{N} \gamma_{i} Q_{i}-I\left(f(\theta)+\sum_{i}^{N} Q_{i}\right)\right)+\lambda(\overline{E S}-E S(Q, \theta)),
$$

where $\mu$ is the shadow cost of the emissions intensity standard $I$. We first consider the "traditional" emissions intensity standard that ignores energy efficiency, such that $f(\theta)=0$ in equation 3. Analysis of this problem leads to our first proposition regarding emission intensity standards.

Proposition 1. Under inelastic demand, the emissions intensity standard without crediting cannot recover the first-best allocation, and the resulting allocation is equivalent to that achieved under a combined emissions tax and energy efficiency tax.

There are two key effects of the emissions intensity standard. First, it acts as an implicit tax of magnitude $\mu \gamma_{i}$ that penalizes higher-emitting sources. Second, it provides a subsidy

${ }^{8}$ Note that the emissions tax does not directly affect the efficiency sector. Rather, by raising the marginal costs of generation, it induces a substitution to efficiency to meet the fixed energy services demand. 
to all forms of electricity generation via $\mu I$. Further manipulation shows that:

$$
\frac{c_{i}\left(Q_{i}\right)+\mu \gamma_{i}-\mu I}{\partial E S / \partial Q}=\frac{c_{j}\left(Q_{j}\right)+\mu \gamma_{j}-\mu I}{\partial E S / \partial Q}=\frac{d e / d \theta}{\partial E S / \partial \theta} \quad \forall i, j
$$

For generation technologies with emission rates in excess of the standard $\gamma_{i}>I$ (e.g. coal), the standard is a net tax, while for technologies with emission rates less than the standard $\gamma_{i}<0$ but potentially greater than zero (e.g. natural gas), the standard is a net subsidy. Thus, per Proposition 1, even if the standard is set such that $\mu=\tau$, it is impossible to recover first-best due to the presence of the term $\mu I .^{9}$

Furthermore, comparing equation 9 with 7 , the presence of the the term $\mu I$ implies too little investment in energy efficiency. While others have noted the inefficiency of standards in the energy sector due to $\mu I$, the above results directly show that the output subsidy for the energy sector acts as an implicit relative tax on energy efficiency. In fact, as Proposition 1 shows, the allocation under the traditional intensity standard is equivalent to that under an emissions tax coupled with a tax on energy efficiency. Given that increasing energy efficiency is one of the "pillars" of the proposed Clean Power Plan, clearly a traditional emissions intensity standard is problematic. While an additional instrument (in this case an electricity tax) or modification of the intensity-coefficients could eliminate this output subsidy and recover first-best, we next examine whether or not a simple adjustment to the standard itself can achieve first-best.

\footnotetext{
${ }^{9}$ If coal is a high-emitting source and gas is a low-emitting source, then the emission intensity standard acts like an implicit tax on coal, inducing substitution out of coal, but it also generates an implicit subsidy to gas. This is obviously inefficient as gas generates emissions and should be taxed. This is the essence of the output subsidy effect noted by other authors, and indeed is one of the general criticism of the use of standards (Helfand 1991; Holland, Hughes, and Knittel 2009; Fullerton and Heutel 2010).
} 
Now suppose the regulator credits energy efficiency as a zero-emission generator according to the rule $f(\theta)$ in the denominator of equation 3. This leads to the following proposition regarding the implications of crediting when demand for energy services is inelastic:

Proposition 2. Under inelastic demand, crediting creates an implicit subsidy to energy efficiency. Crediting at the rate $f^{\prime}(\theta)=\frac{\partial E S / \partial \theta}{\partial E S / \partial Q}$ and setting the standard such that $\mu=\tau$ recovers the first-best allocation.

The emissions intensity standard with efficiency crediting has a number of similarities with the traditional intensity standard, but with the key difference that it introduces a subsidy $\mu I f^{\prime}(\theta)$ to the energy efficiency sector:

$$
\frac{c_{i}\left(Q_{i}\right)+\mu \gamma_{i}-\mu I}{\partial E S / \partial Q}=\frac{c_{j}\left(Q_{j}\right)+\mu \gamma_{j}-\mu I}{\partial E S / \partial Q}=\frac{d e / d \theta-\mu I f^{\prime}(\theta)}{\partial E S / \partial \theta} \quad \forall i, j .
$$

Per Proposition 2, setting crediting at $f^{\prime}(\theta)=\frac{\partial E S / \partial \theta}{\partial E S / \partial Q}$ and the standard such that $\mu=\tau$ recovers the first-best allocation. ${ }^{10}$ Intuitively, the implicit subsidy to energy efficiency offsets the output subsidy to generation, removing the relative distortion between generation and energy efficiency. Furthermore, because $\overline{E S}=E S(Q, \theta)$, the resulting allocation for both generation and energy efficiency from the emissions intensity standard with efficiency crediting is equivalent to the first-best emissions tax.

Focusing further on the emissions crediting rule of $f^{\prime}(\theta)=\frac{\partial E S / \partial \theta}{\partial E S / \partial Q}$, in the simple (and plausible) case where energy consumption and energy efficiency are perfect substitutes $(E S=$ $Q+\theta$ ), then one-for-one crediting whereby $f(\theta)=\theta$ achieves first-best. If energy services are

${ }^{10}$ The ratio $\frac{\partial E S / \partial \theta}{\partial E S / \partial Q}$ is the marginal rate of technical substitution between energy efficiency and consumption in the household's production function for energy services. 
a more complicated non-linear function of generation and energy efficiency, the crediting rule becomes more complicated, but nonetheless a first-best crediting rule exists. Thus, rather than introduce an additional instrument to recover first-best, crediting energy efficiency in the denominator of the standard creates an implicit subsidy for output reduction, offsetting the output subsidy effect and achieving first-best outcomes.

\subsection{Elastic demand for energy services}

Analyzing emissions intensity standards when demand for energy services is downwardsloping requires a few modifications to the above framework to account for changes in consumer surplus arising from changes in energy services. Denote the inverse demand for energy services as:

$$
P(E S(Q, \theta))
$$

which satisfies $P^{\prime}<0$. For all models considered below, a representative household chooses energy consumption and energy efficiency to maximize the area under the demand curve net of expenditures:

$$
\max _{Q, \theta} \int_{0}^{E S(Q, \theta)} P(q) d q-P_{E} Q-P_{\theta} \theta
$$

where $P_{E}$ is the price of electricity and $P_{\theta}$ is the price of energy efficiency. 


\subsubsection{First-best emissions tax}

Under a first-best emissions tax $\tau$ equal to the marginal external damage of emissions, the representative firm maximizes profits by choosing generation and energy efficiency per:

$$
\max _{Q_{i}, \theta} P_{E} \sum_{i}^{N} Q_{i}+P_{\theta} \theta-\sum_{i}^{N} \int_{0}^{Q_{i}} c_{i}\left(q_{i}\right) d q_{i}-e(\theta)-\tau\left(\sum_{i}^{N} \gamma_{i} Q_{i}\right)
$$

whereby the firm equates marginal generation costs inclusive of emissions damages to the price of electricity and equates the marginal cost of efficiency to the price of energy efficiency. Combining with the households problem yields the following intuitive lemma regarding optimal regulation under elastic demand:

Lemma 2. Under elastic demand, the first-best allocation equates marginal private benefits of generation and energy efficiency with the marginal social costs.

$$
\begin{aligned}
& P(E S(Q, \theta)) \frac{\partial E S}{\partial Q}=c_{i}\left(Q_{i}\right)+\tau \gamma_{i} \quad \forall i \\
& P(E S(Q, \theta)) \frac{\partial E S}{\partial \theta}=\frac{d e}{d \theta}
\end{aligned}
$$

\subsubsection{Emissions intensity standard with and without crediting}

The preceding derivation yields the resulting allocation under first-best regulation via an emissions tax. We now contrast that allocation with the resulting allocation from emissions intensity standards. Consider a representative firm subject to an emissions intensity standard 
with crediting rule $f(\theta)$. The firm's problem is then:

$$
\max _{Q_{i}, \theta} P_{E} \sum_{i}^{N} Q_{i}+P_{\theta} \theta-\sum_{i}^{N} \int_{0}^{Q_{i}} c_{i}\left(q_{i}\right) d q_{i}-e(\theta)+\mu\left(I\left(f(\theta)+\sum_{i}^{N} Q_{i}\right)-\sum_{i}^{N} \gamma_{i} Q_{i}\right) .
$$

We first consider the traditional emissions intensity standard, whereby $f(\theta)=0$ in equation 3. Examining the solution to the above in the absence of crediting gives rise to the following proposition:

Proposition 3. Under elastic demand, the emissions intensity standard can not recover first-best. If the standard is set such that $\mu=\tau$, energy services are underpriced, leading to an underprovision of energy efficiency.

As in the inelastic demand case, the traditional emissions intensity standard does not yield the first-best allocation due to the presence of the subsidy $\mu I$. Comparing again between the electricity sector and the energy efficiency sector, the output subsidy effect distorts the relative incentives, favoring generation at the expense of energy efficiency. This implicit subsidy to generation lowers the price of energy services, leading to less incentive for households to purchase energy efficiency.

In the inelastic demand case, crediting energy efficiency via $f(\theta)$ in the denominator of the standard could potentially recover first-best. Examining the effects of crediting in the elastic demand case leads to the following proposition:

Proposition 4. Under elastic demand, the emissions intensity standard with crediting cannot recover first-best. Crediting at $f^{\prime}(\theta)=\frac{\partial E S / \partial \theta}{\partial E S / \partial Q}$ offsets the output subsidy effect, but creates an energy services subsidy effect. 
Again, including crediting within the standard generates an implicit subsidy to energy efficiency equal to $\mu I f^{\prime}(\theta)$. Per Proposition 4 the crediting rule $f^{\prime}(\theta)=\frac{\partial E S / \partial \theta}{\partial E S / \partial Q}$ removes the relative distortion created by the output subsidy effect as all sectors receive the same subsidy per unit of energy service. However, in contrast to the case of inelastic demand, when demand for energy services is downward sloping, crediting energy efficiency is no longer first-best due to the demand response via $P(E S(Q, \theta))$. While crediting energy efficiency removes the output subsidy effect that favors generation, it creates its own distortion - an energy services subsidy effect - that leads to too large of energy services consumed relative to first-best. This suggests there is a tradeoff to crediting energy efficiency in the sense that too little crediting leads to a relative distortion between generation and efficiency, while too much crediting distorts the overall level of energy services. We return to this point analytically in the next section, and in the numerical application to follow.

\subsection{Optimal crediting rule}

The above results indicate that selecting the crediting rule is an important regulatory consideration. For example, under elastic demand for energy services, an important tradeoff emerges - while crediting energy efficiency offsets the relative distortion created by the output subsidy effect, it creates an absolute distortion in the level of energy services consumed. Suppose a regulator was to implement the crediting rule $f(\theta)=\kappa \theta$, whereby energy efficiency is credited at some constant rate, such that $f^{\prime}(\theta)=\kappa$. What crediting rule $\kappa$ should the regulator select, conditional on understanding how the decentralized market will respond to 
a given standard $I$ ? Formally, the regulator solves:

$$
\max _{\kappa} W=\int_{0}^{E S(Q(\kappa), \theta(\kappa))} P(q) d q-\sum_{i}^{N} \int_{0}^{Q_{i}(\kappa)} c_{i}\left(q_{i}\right) d q_{i}-e(\theta(\kappa))-\tau\left(\sum_{i}^{N} \gamma_{i} Q_{i}(\kappa)\right),
$$

where $\tau$ is the marginal social damage from emissions.

Proposition 5. The optimal crediting rule $\kappa$ balances the deadweight loss from distorting the efficiency sector with the deadweight loss from the electricity sector.

$$
(\mu I \kappa) \frac{d \theta}{d \kappa}=\sum_{i}^{N}\left[\left(\mu\left(\gamma_{i}-I\right)-\tau \gamma_{i}\right) \frac{d Q_{i}}{d \kappa}\right]
$$

The left term is the welfare loss from distorting the energy efficiency market via crediting, while the right side is the welfare losses associated with the changes in the output subsidy distortions due to crediting on the generation side. We now consider the setting of the optimal crediting rule $\kappa$ under the inelastic and elastic demand cases.

\subsubsection{Inelastic demand for energy services}

First, consider the case of optimal crediting under inelastic demand:

Proposition 6. Under inelastic demand, the optimal crediting rule is $\kappa=\frac{\partial E S / \partial \theta}{\partial E S / \partial Q}$ if and only if the standard is set such that $\mu=\tau$. No other combination of $\kappa$ and $\mu$ can be optimal.

Combining the results in Proposition 6 with those from Section 2.1 yields several important policy insights. Consider the abatement costs of the emissions intensity standard relative to the abatement costs of an emissions tax. The above implies that under inelastic 
demand, setting the crediting rule $\kappa=\frac{\partial E S / \partial \theta}{\partial E S / \partial Q}$ and corresponding standard will yield a relative abatement cost ratio of 1 . Furthermore, it implies that any deviation from this optimal $\kappa$ and/or $\mu$ will lower welfare/raise abatement costs, such that the relative abatement cost of the emissions intensity standard will grow as the crediting rule deviates further from the optimal rule. ${ }^{11}$ Thus, relative abatement costs as a function of the crediting rule $\kappa$ should be U-shaped with a minimum occurring at $\kappa=\frac{\partial E S / \partial \theta}{\partial E S / \partial Q}$, whereby abatement costs of the emissions intensity standard exactly equal those under an emissions tax.

\subsubsection{Elastic demand for energy services}

Turning now to the case of elastic demand for energy services:

Proposition 7. Under elastic demand, if the crediting rule is set $\kappa=\frac{\partial E S / \partial \theta}{\partial E S / \partial Q}$, then $\mu<\tau$ if and only if emissions are declining as crediting increases $\left(\sum_{i}^{N}\left[\gamma_{i} \frac{d Q_{i}}{d \kappa}\right]<0\right)$. If the standard is set such that $\mu=\tau$, then $\kappa<\frac{\partial E S / \partial \theta}{\partial E S / \partial Q}$ if and only if generation declines as crediting increases $\left(\sum_{i}^{N}\left[\frac{d Q_{i}}{d \kappa}\right]<0\right)$. The optimal $\kappa$ implicitly satisfies:

$$
\kappa=\frac{\sum_{i}^{N}\left[\frac{d Q_{i}}{d \kappa}\right]}{\frac{\partial E S / \partial Q}{\partial E S / \partial \theta} \sum_{i}^{N}\left[\frac{d Q_{i}}{d \kappa}\right]-\frac{d E S}{d \kappa}}
$$

Proposition 7 reveals several important points regarding the tradeoff between the relative and absolute distortions associated with intensity standards and elastic demand. If the crediting rule is that $\kappa=\frac{\partial E S / \partial \theta}{\partial E S / \partial Q}$, then the standard should be set such that the shadow

11 The intuition is that by setting the "wrong" standard and thus shadow cost $\mu$, the generation sector is distorted in a way that can never be fully compensated by changing the efficiency crediting rule. Similarly, by setting the wrong crediting rule, changing the standard can strike the right balance between efficiency and the generation sectors, but at the cost of distorting the relative incentives within the generation sector. 
price of the standard is less than the marginal social damage/emissions tax. Conversely, if the standard is set such that $\mu=\tau$, then intuitively as the energy services demand response $d E S / d \kappa$ gets larger, the optimal crediting ratio is cranked down as the absolute distortion begins to outweigh the relative distortion. ${ }^{12}$

The above results plus the findings in Section 2.2 again have important policy implications. While the optimal crediting rule implicitly defined in equation 18 generates the greatest welfare under an emissions intensity standard, from Section 2.2 that crediting rule does not provide an identical allocation to that under an emissions tax. As such, the relative abatement cost of the emissions intensity standard will be minimized at the optimal crediting rule, but because of the elastic demand response, the abatement cost of the emissions intensity standard will always exceed that under the emissions tax.

\section{$3 \quad$ Numerical exercise}

In this section, we develop a calibrated numerical model to further explore the analytic results presented above. In particular, we examine the relative efficiency of alternative energy efficiency crediting levels with respect to an emissions tax in a model that incorporates a much more detailed depiction of the electricity dispatch problem. As discussed further below, many of the modeling choices that follow are motivated by the features of the Clean Power Plan. ${ }^{13}$

\footnotetext{
12 Note that in equation 18, as energy service demand becomes more inelastic ( $\frac{d E S}{d \kappa}$ goes to zero), the optimal crediting rule converges to $\kappa=\frac{\partial E S / \partial \theta}{\partial E S / \partial Q}$.

${ }^{13}$ In particular, because states are given the option to adopt mass-based policies such as cap and trade (akin to an emissions tax), understanding the relative efficiency of the rate-based emissions standard approach is important.
} 
The model is calibrated to represent a specific National Energy Reliability Corporation (NERC) region, the Electric Reliability Council of Texas (ERCOT) region. We choose this region for several reasons. First, this transmission region of the U.S. has relatively small electricity import/export capacities, so modeling it as a closed system is not an egregious over-simplification as it may be for other regions. Second, the ERCOT region represents about 90 percent of the load in Texas. As the CPP sets state-level targets, calibrating the model to ERCOT effectively allows us to analyze a specific state's response to a CPP-like policy.

To analyze emission tax and intensity standard policies in a dispatch model that incorporates energy efficiency, we first must specify a demand for energy services $(E S)$. We assume a constant-elasticity demand of the form:

$$
E S_{h}\left({ }_{h} P\right)=\phi_{h} P_{h}^{\epsilon}
$$

where $E S_{h}$ is the demand for energy services in time period (hour) $h, P_{h}$ is the implied unitcost of energy services, $\phi_{h}$ is a scale parameter, and $\epsilon$ is the elasticity of demand for energy services. The unit-cost formulation for energy services embeds the optimizing behavior by households and in CES form is given by:

$$
P_{h}\left(P_{E h}, P_{\theta h}\right)=\left(\alpha^{\sigma} P_{\theta h}^{1-\sigma}+(1-\alpha)^{\sigma} P_{E h}^{1-\sigma}\right)^{1 /(1-\sigma)}
$$

where $P_{E h}$ is the price of electricity in hour $h, P_{\theta h}$ is the price of energy efficiency in hour $h$, $\alpha$ is the productivity of energy efficiency in the production of energy services and $\sigma$ is the 
elasticity of substitution between electricity $(Q)$ and energy efficiency $(\theta)$. Applying Shephard's Lemma on the expenditure function $E S_{h}\left(P_{h}\right) * P_{h}\left(P_{E h}, P_{\theta h}\right)$ yields the hourly demand functions for electricity, $Q_{h}\left(P_{E h}, P_{\theta h}\right)$, and energy efficiency, $\theta_{h}\left(P_{E h}, P_{\theta h}\right)$, as a function of prices $P_{E h}$ and $P_{\theta h}$ as:

$$
\begin{gathered}
Q_{h}\left(P_{E h}, P_{\theta h}\right)=\phi_{h} P_{E h}^{-\sigma}(1-\alpha)^{\sigma}\left(P_{\theta h}^{(1-\sigma)} \alpha^{\sigma}+P_{E h}^{(1-\sigma)}(1-\alpha)^{\sigma}\right)^{\left(\frac{\epsilon+\sigma}{1-\sigma}\right)} \\
\theta_{h}\left(P_{E h}, P_{\theta h}\right)=\phi_{h} P_{\theta h}^{-\sigma} \alpha^{\sigma}\left(P_{\theta h}^{(1-\sigma)} \alpha^{\sigma}+P_{E h}^{(1-\sigma)}(1-\alpha)^{\sigma}\right)^{\left(\frac{\epsilon+\sigma}{1-\sigma}\right)}
\end{gathered}
$$

Note that these functional forms assume that the energy efficiency market clears hourly and depends on hourly electricity prices. We chose this specification for our base policy comparison results because it is closer in spirit to the static analytical model developed above where electricity and energy efficiency markets clear contemporaneously for the given period. In reality, it is likely that energy efficiency would, in part, be the result of durable purchases and thus not necessarily respond to hourly fluctuations in electricity prices. That said, energy-efficiency providing durables would still provide time-varying energy savings (MWh's avoided). More specifically it would seem reasonable that these durables would provide more energy savings in high electricity demand periods and less in low demand periods. ${ }^{14}$ In that respect, the energy efficiency demand function provided above, $\theta_{h}\left(P_{E h}, P_{\theta h}\right)$, would roughly approximate this market outcome as $\theta_{h}$ responds positively to increases in $P_{E h}$ and $\phi_{h}$, both of which increase in periods of high electricity demand.

For other parameters of note, we assume constant values for $\alpha, \sigma$, and $\epsilon$ (summarized

\footnotetext{
${ }^{14}$ For example, if energy efficiency is provided through more energy efficient air conditioning units, those units would provide more energy savings during hot time periods (i.e. high demand periods) than in hours of moderate temperature (i.e. low demand periods).
} 
in Table 1), though we also vary these in sensitivity analyses. We also assume a constant marginal cost of energy efficiency, $c_{\theta}$, and thus in the absence of additional energy efficiency policies $c_{\theta}=P_{\theta h} \forall h .^{15} \quad$ We then use these assumed values, along with observed 2013 hourly electricity prices and quantities for ERCOT to derive hourly values for the remaining parameter, $\phi_{h}{ }^{16}$

We next form hourly electricity supply curves (the dispatch curve) and an energy efficiency curve to solve the market equilibriums. The energy efficiency supply curve is formed via the assumed constant marginal cost for energy efficiency, $c_{\theta}$. For the electricity supply curve, we begin by collecting data on heat rates (MMBtu of fuel burned per MWh of generation), non-fuel variable operation and maintenance costs (VOM, given in $\$ / M W h$ ), capacity, scheduled outage rates, and forced outage rates for all non-renewable generating facilities in ERCOT in 2013. Using fuel prices for coal, natural gas, and uranium from the EIA's Annual Energy Outlook (AEO) 2014, along with heat rates and VOM's, we form a marginal generation cost for each generator. ${ }^{17}$

15 The assumed value of the marginal cost of energy efficiency is taken from Arimura et al. (2012). The approximation of a constant marginal cost for energy efficiency is likely not limiting over a smaller range of energy efficiency levels, but may be more problematic if simulated energy efficiency levels differ considerably from current levels. However, we maintain a constant marginal cost assumption for the entirety of this analysis as it dramatically reduces the complexity of deriving equilibrium values. We also vary this constant value in our sensitivity analysis.

16 This price and quantity data, as well as all other generator specific and wind generation data discussed below, was downloaded from the data management firm ABB (formerly Ventyx), which collects and organizes publicly available data on the electricity sector. The underlying data is available from EIA-860 forms, EPA's Continuous Emissions Monitoring System, and ERCOT's website. For ERCOT electricity prices and quantities were given for four sub-regions: South, West, North, Houston. We create a single load variable by summing each sub-region's hourly quantity and create a single quantity-weighted price for all of ERCOT to calculate the $\phi$ parameter.

${ }^{17}$ For generator $i$, marginal cost is given as $c_{i}=h r_{i} P_{i}^{f}+V O M_{i}$ where $h r_{i}$ is $i$ 's heat rate and $P_{i}^{f}$ is its fuel price. For semi-nonrenewable generation plants, such as those fueled by 
To account for the impacts of forced and scheduled outage rates, we effectively reduce the capacity of each non-renewable plant that is available for generation. The forced and scheduled outage rates are given as the percent of annual hours for which the plant is closed down. We follow the procedure of Fell and Linn (2013) to account for these outage rates. More specifically, we first assume that scheduled maintenance occurs in ERCOT's low-demand months: February - April and October - December. We then reduce the maximum possible capacity factors by a constant rate over these months, such that the average capacity factor across all hours of the year for each generator matches one minus the reported average scheduled outage rate. For the closures due to unscheduled maintenance on the nonrenewable generators, we assume that in each hour of the year, including those in the assumed scheduled maintenance period, the capacity factor is lowered by a constant rate such that the cumulative reduction in the capacity factor is equal to the average unscheduled maintenance rate. Multiplying these outage-rate adjusted maximum possible capacity factor by each plant's given capacity, we then have the effective capacity available to generate for each plant for each hour of the year.

Also, similar to Fell and Linn (2013), we assume coal and natural gas combined cycle (NGCC) plants have limited ramping capabilities, which we model simplistically as a minimum "must-run" constraint. For coal plants we assume that they must run at a minimum of 40 percent of their given capacity and that NGCC plants must run at a minimum of 15 percent of their capacities. ${ }^{18}$ landfill gases, we assume a fuel price of zero.

${ }^{18}$ Fell and Linn (2013) use must-run limits of 40 and 30 percent of capacity for coal and NGCC plants, respectfully. Upon examination of the actual hourly generation data for 2013 , the 30 percent must-run constraint appears too strict. We therefore reduced it to 15 percent, which is approximately the fifth percentile capacity factor among NGCC plants in ERCOT, 
Finally, we collect data on hourly wind generation and effectively model wind generation as a single generator, treating it as a zero marginal cost generation source. ${ }^{19}$ Combining this wind generation with the marginal costs of the non-renewable sources and their hourly effective capacity, we form the hourly supply curve by ordering generators from lowest to highest marginal costs. Given the hourly electricity supply curve, assumed constant marginal cost of energy efficiency and the demand equations 21 and 22, we can calculate the hourly market clearing conditions (prices and quantities) for electricity, energy efficiency, and energy services as a whole. ${ }^{20}$

\subsection{Policy Comparison}

The goal of our policy comparison is to calculate the cost of achieving a given emissions level under an emissions standard regulation, with and without energy efficiency crediting, as compared to the cost of achieving the same emissions level under an emissions tax. We assume the policies take place in 2020 and therefore use the AEO 2014 projected fuel prices for ERCOT for that year, though we do assume that generation capacity remains fixed. ${ }^{21}$ conditioned on those plants having positive generation.

${ }^{19}$ Modeling all wind farms as a single generator abstracts from local concentrations of wind generation and resulting local transmission congestion - modeling transmission constraint issues is beyond the scope of this simulation exercise. Additionally, generation from solar plants are excluded as they make up a very small fraction of generation in ERCOT.

${ }^{20}$ The simulation technique used here does assume hourly energy efficiency decisions. This, in many instances, is a more flexible form of energy efficiency updating than is likely possible for many technologies. However, more restrictive energy efficiency updating forms also impose some assumptions that may not be relevant, so we opt for the most flexible form for our framework.

${ }^{21}$ The first interim policy goals for the CPP take place between 2020 - 2030, so we opted for 2020 as the year the policy takes effect. AEO 2014 projections for the Texas region do show some expansion in renewable and NGCC generation capacity in a reference case that does not include the CPP. Capacity of the remaining generating sources remained relatively flat from 2013 - 2020. Given this and the added complexity, both computationally and in terms 
For the standards model with energy efficiency crediting, we assume a simple linear crediting function such that $f(\theta)=\kappa \theta$ where $\kappa \geq 0$. Given this assumption about energy efficiency crediting the effective emissions rate is calculated:

$$
I=\frac{\sum_{h=1}^{H} \sum_{i=1}^{N} \gamma_{i} Q_{i h}}{\sum_{h=1}^{H} \sum_{i=1}^{N} Q_{i h}+\sum_{h=1}^{H} \kappa \theta_{h}}=\frac{\text { Emissions }}{Q+\kappa \theta}
$$

In practice, emission standard compliance will be achieved via a "tradable performance standard" (TPS) system, whereby generators with emission rates above the standard will purchase credits from generators with below-standard emission rates. Associated with this resulting TPS market is a price for the credits, represented as $\mu$ in the analytic modeling above. This price for credits affects the marginal costs of generators, such that $c_{i}=h r_{i} P_{i}^{f}+$ $V O M_{i}+\mu\left(\gamma_{i}-I\right)$ and the effective price of energy efficiency such that $P_{\theta h}^{T P S} \equiv P_{\theta}^{T P S}=$ $c_{\theta}-\kappa \mu I{ }^{22}$ To find the emissions standard that achieves the target emissions level, we use an iterative search process. The process begins by giving the dispatch with energy efficiency model a certain emissions standard. The model then searches for a TPS credit price, $\mu$, that makes the standard just binding. The model then compares the emissions from the binding standard to the target level and continues to feed the model values of the standard until the difference between the binding-standard emissions meets that of the target. ${ }^{23}$

Finding the emissions tax, $\tau$, that leads to an emissions level that hits the target is of interpreting results, we do not include a capacity expansion component to our model.

${ }^{22}$ We assume an emissions intensity for natural gas as $116.9 \mathrm{lbs} / \mathrm{MMbtu}$ and for coal of 210.6lbs/MMBtu based on EPA calculated averages given at http://www.epa.gov/ cleanenergy/energy-resources/refs.html.

${ }^{23}$ This is operationalized as a minimization problem, minimizing the difference in emissions from the binding standard and the emissions target, in Matlab using the solver "fminunc". We use a similar process for the minimization problem to find the emissions tax rate. 
done in a similar, though somewhat simpler manner. The tax also alters the marginal cost of generators, such that $c_{i}=h r_{i} * P^{f}+V O M_{i}+\tau \gamma_{i}{ }^{24} \quad$ The search is again set up as a minimization problem where the solver finds the tax rate that minimizes the difference between the resulting emissions under the tax and the target emission level.

The cost of meeting the target emissions level under a given policy $j$, with $j=[$ TPS, Tax $]$, has several components and is given relative to the "no emissions policy" baseline. More specifically, we calculate the cost of complying with the target via policy $j$ as:

$$
C^{j}=\sum_{h=1}^{H}\left(\int_{E S_{h}^{j}}^{E S_{h}^{0}} P\left(E S_{h}\right) d E S\right)-\sum_{i=1}^{N} \sum_{h=1}^{H}\left(c_{i}\left(Q_{i h}^{0}-Q_{i h}^{j}\right)+\tau \gamma_{i} Q_{i h}^{j}\right)-\sum_{h=1}^{H} c_{\theta}\left(\theta_{h}^{0}-\theta_{h}^{j}\right),
$$

where $E S_{h}^{0}$ is the baseline energy services in hour $h, E S_{h}^{j}$ is energy services under policy $j$, $P\left(E S_{h}\right)$ is the inverse demand for energy services, $\left(Q_{i h}^{0}-Q_{i h}^{j}\right)$ is the difference in hourly generation from generator $i$ between the baseline and policy $j$, and $\left(\theta_{h}^{0}-\theta_{h}^{j}\right)$ is the difference in energy efficiency under the baseline and policy $j$. Thus, the first term of equation 24 measures the change in ES total consumer surplus (area under the ES demand curve) from implementing the policy. The second term accounts for the change in the generation costs, with the assumption that if an emissions tax is imposed tax revenues are returned to consumers in a lump-sum fashion. The final term accounts for the value of the change in energy efficiency purchased. To put this cost value into a more interpretable measure, we report the average annual cost per unit of emissions abated, $(C / A)^{j}=C^{j} /\left(\right.$ Emissions $^{0}-$ Emissions $\left.^{j}\right)$.

\footnotetext{
${ }^{24}$ Note that the emissions tax also indirectly affects energy efficiency through the price of electricity.
} 


\subsection{Simulation Results}

Reference case parameter policy comparisons are given in Table 2. This table gives the results from a TPS and emissions tax policy aimed at reducing emissions 20 percent below the predicted "no policy" baseline of $2020 \mathrm{CO}_{2}$ emissions, a target in line with the CPP's goal for Texas. ${ }^{25}$ For the TPS policy, we consider different energy efficiency crediting rates, $\kappa$, ranging from 0 to 1.2. The bottom two lines of Table 2 give the summary results from the tax and baseline runs, which are unaffected by the assumed energy efficiency credit rates. The remainder of the results are for the TPS outcomes.

Of primary importance from an efficiency standpoint are the "Cost Ratio" outcomes. This row gives the ratio of the TPS's to tax's average policy cost per unit of abatement (ratio of $(C / A)^{T P S}$ to $(C / A)^{T a x}$ ), and can thus be seen as a measure of relative efficiency. As is mentioned above, the relative inefficiency of the TPS to tax policies is U-shaped over the range of crediting rates explored, with the inefficiency minimized at a crediting rate near 0.8. This, of course, is not a general result and will vary with assumed parameterizations as we show in more detail below.

Remaining results in Table 2 are largely as expected. With increasing energy efficiency crediting, the standard, $I$, and related TPS credit price, $\mu$, needed to meet the emissions target are falling. This is as expected because increasing the crediting rate subsidizes energy efficiency, increasing energy efficiency levels, $\theta^{T P S}$, and increasing the denominator of the standard as shown in equation 23. Consequently, increasing $\kappa$ leads to a decrease in electricity consumption, $Q^{T P S}$, and correspondingly lower average electricity prices relative to

\footnotetext{
${ }^{25}$ Projected and target emissions for Texas under the CPP were taken from http://www . epa.gov/airquality/cpptoolbox/texas.pdf.
} 
the tax policy and even relative to the no-policy baseline results. However, as the analytic model predicts, subsidizing energy efficiency leads to an overall increase in energy service levels, $E S^{T P S}$ and relatively too much $E S$ compared to the efficient levels generated by the emissions tax.

\subsection{Sensitivity Analysis}

To further explore how the chosen parameterization has affected our main results and to more fully describe the policy implications of energy efficiency crediting we conduct a variety of sensitivity analyses. We present a summary of these analyses by plotting the "Cost Ratio" under different parameter settings and energy efficiency crediting rates, $\kappa$. These plots are given in Figures 1 and 2. ${ }^{26}$ Before discussing these sensitivity analyses, it is useful to recall how the analytic results show energy efficiency crediting can offset the production subsidy inefficiencies that arise in a typical rate-based standards policy. More specifically, subsidizing energy efficiency through crediting induces a substitution out of electricity consumption, and thus reduces some of the inefficiencies associated with the implicit electricity production subsidy. We can therefore think about how altering a given parameter impacts the cost of energy efficiency relative to electricity production and therefore what level of energy efficiency crediting best reduces the electricity production subsidy. This reasoning is used to explain observed optimal crediting rates in the sensitivity analyses discussed below.

The plots in Figure 1 summarize results from our sensitivity analysis surrounding the parameters in the assumed CES unit cost function given in equation 20. The top panel of Figure 1 plots the Cost Ratio across a range of energy efficiency crediting values for three

\footnotetext{
${ }^{26}$ More complete summaries of the sensitivity analyses are given in Appendix B.
} 
different assumed values of $\alpha{ }^{27}$ Recall $\alpha$ can be thought of as measuring the productivity of energy efficiency in creating energy services, where a higher $\alpha$ implies the energy efficiency investments are relatively better at providing energy services. ${ }^{28}$ Correspondingly, our sensitivity analysis shows that at the relatively high $\alpha=0.8$ value, the Cost Ratio is minimized (over the values explored) at a low $\kappa=0.2$. We would expect that a higher $\alpha$ would lead to a lower optimal $\kappa$ - if the energy efficiency investment is more productive at creating energy services, then there will already be relatively high investment in energy efficiency and only a small amount of subsidizing is needed to get to the optimal TPS policy. Furthermore, with higher values of $\alpha$, high levels of energy efficiency crediting can create inefficiently high levels of total energy services and further distort the TPS relative to the emissions tax. Indeed, results under the parameterization of $\alpha=0.8$ imply that no crediting, $\kappa=0$, would create less of a distortion relative to the efficient emissions tax policy than credit rates near 1. However, as we decrease $\alpha(\alpha=0.4$ or $\alpha=0.2)$, our results indicate that optimal energy efficiency crediting rates would be larger. Finally, note that with a very small $\alpha$, such as our case with $\alpha=0.2$, varying the crediting rate for energy efficiency has little impact on the

${ }^{27}$ Note that for every $\alpha$ considered, as well as for other demand parameters considered, the $\phi$ values are recalculated, again calibrated using observed 2013 electricity prices and quantities. All other parameters not mentioned remain the same as in the reference case and that is also true in our other parameterizations examined. Also, for all sensitivity analyses, we have aggregated from the hourly frequency to the 12-hour frequency. We found this aggregation had little numerical impacts on the results and greatly reduced computation times. In addition, we explored numeric models that used even more aggregated timeperiods (smaller $H$ ) and the central comparison of TPS and tax policies remains similar to the results shown here, giving us additional confidence that our hourly modeling construct for energy efficiency is not unduly influencing our results.

${ }^{28}$ For example, if light from an energy efficient bulb is viewed by consumers to be as "good" as or "better" than light from an incandescent bulb, $\alpha$ would be relatively high for that case. But, if the light from the energy efficient bulb is considered "inferior" then it would imply a relatively low $\alpha$ value. 
relative efficiency of the TPS policy. This is again as expected because a small $\alpha$ implies that energy efficiency is relatively unproductive at generating energy services and will therefore not be invested in even if it is subsidized.

The second panel of Figure 1 explores the sensitivity with respect to $\sigma$, the elasticity of substitution between electricity and energy efficiency. Here we show that across the three values examined, $\sigma=0.8,2.0,3.2$, the optimal crediting value remained relatively stable near 0.8-1.0. However, the relative gains (reduction in relative inefficiency as measured by the Cost Ratio) from crediting are much higher when the degree of substitution is larger. ${ }^{29}$ This makes intuitive sense because when the elasticity of substitution is relatively low, such as our $\sigma=0.8$ case, then the ability to substitute out of electricity to energy efficiency is restricted and thus crediting energy efficiency will not illicit much of an increase in energy efficiency investment.

The bottom panel of Figure 1 examines the sensitivity with respect to the energy service price elasticity parameter, $\epsilon$. This parameter directly relates to our analytic section above as we show that for the case of perfectly inelastic energy services demand, the TPS can be as efficient as an emissions tax. Correspondingly, our simulation results with near perfectly inelastic energy services demand, $\epsilon=1.0 \mathrm{E}^{-10}$, has a cost ratio of approximately one at the crediting rate of $\kappa=1 .^{30}$ However, when energy service demand elasticity becomes much greater, for example at $\epsilon=-0.4$, subsidizing energy efficiency by increasing $\kappa$ does un-do

${ }^{29}$ Under the cases where $\sigma=2$ and $\sigma=3.2$ the cost ratio falls from about 1.4 with $\kappa=0$ to around 1.1 with $\kappa=1$, whereas with $\sigma=0.8$ the cost ratio only varies from about 1.25 to 1.1 .

30 Note that with inelastic demand for energy services, an optimal $\kappa=1$ is not a general result, but rather is specific to this simulation exercise. In other words, the marginal rate of technical substitution in this parameterization happens to be such that the optimal $\kappa=1$. 
some of the production subsidies implicit in a TPS, but also induces relatively too much energy efficiency investment. As a result, larger (in magnitude) elasticity measures imply lower optimal crediting rates. The results also show that with larger elasticity values, the TPS is considerably less efficient relative to the emissions tax as noted by the $\epsilon=-0.4$ case having a cost ratio that is considerably higher than the other elasticity cases.

The plots in Figure 2 explore the sensitivity of our results to assumptions about market conditions and policy stringency by varying the assumed marginal cost of energy efficiency $\left(c_{\theta}\right), 2020$ expected natural gas prices $\left(P_{N G}\right)$, and the level of emission reductions. The top panel of Figure 2 displays the cost ratio results across various crediting rates for energy efficiency prices of $\$ 35, \$ 50$, and $\$ 65 /$ MWh avoided. From these plots, we show that that at lower energy efficiency $\operatorname{costs}\left(c_{\theta}=35\right)$ the crediting rate that minimizes the relative inefficiency of the TPS program is at a lower level than it is for cases with higher energy efficiency costs. This is as expected because lower energy efficiency costs will induce a significant amount of energy efficiency adoption regardless of crediting and therefore only a small level of crediting is needed to negate the electricity production subsidy.

The middle panel of Figure 2 varies the assumed price of natural gas. By varying gas prices we alter average electricity prices and emission levels because lower gas prices lead to gas generation displacing coal generation. The low gas price scenario and the baseline gas price assumption $\left(P_{N G}=\$ 3.17 / M M B t u\right.$ and $P_{N G}=\$ 5.17 / M M B t u$, respectively) have similarly shaped curves and both have optimal credit rates near one. The relative inefficiency of the TPS under the low gas price case is, percentage wise, generally quite large. This occurs because with low gas prices, electricity prices are also lower, which lowers incentives for energy efficiency investment. It then becomes difficult to subsidize energy efficiency at 
a rate that is sufficient to un-do the electricity production subsidy component of the TPS. However, in the high natural gas price scenario the optimal credit rate is much smaller, with the $P_{N G}=\$ 7.17 / M M B t u$ case leading to an optimal credit rate near 0.4. We would expect this as higher gas prices lead to higher electricity prices, incentivizing more investment in energy efficiency and reducing the need for energy efficiency crediting.

The bottom panel of Figure 2 plots Cost Ratios under various emission reduction targets, ranging from 10 - 40 percent reductions. One noticeable aspect from this plot is that when the emission reduction target is low, crediting energy efficiency at low levels leads the TPS to be quite inefficient relative to the emissions tax system. This again can be traced back to the electricity prices. With low abatement targets, electricity prices under the TPS are quite low and the energy efficiency levels are much lower than under the tax achieving the same level of emissions. ${ }^{31}$ Only by crediting energy efficiency at higher rates is there a sufficient incentive to invest in energy efficiency. However, the relative inefficiency for the low reductions case remains quite high because increasing the subsidy for energy efficiency not only reduces the price of energy efficiency, but also further drops electricity prices, creating a situation of relatively high energy services consumption regardless of the crediting rate. The other readily observable feature of the emissions reduction comparison is that when emissions reductions are relatively high (reduction $=40 \%$ ) almost all the gains from crediting can be achieved at rather modest crediting rates and that the relative efficiency remains approximately constant across crediting rate levels. This is as expected because with large abatement targets, electricity prices even under the TPS are quite high. This

\footnotetext{
${ }^{31}$ With no energy efficiency crediting, the TPS average electricity price was $\$ 38.57 / \mathrm{MWh}$ compared to an emissions-unconstrained price of $\$ 37.36$ and price under the tax of $\$ 42.34$.
} 
high price sufficiently restricts electricity consumption and also incentivizes energy efficiency adoption such that increasing the crediting rate has little impact on the level of adoption.

\section{Discussion}

Under the EPA's CPP, both mass-based (cap and trade) and rate-based (emission intensity standards) policies can be used by states to achieve compliance with their targets. ${ }^{32}$ Despite the fact that, from a national welfare perspective, rate-based policies may be a secondbest alternative to optimal mass-based policies, many states may favor rate-based policies as an option for CPP compliance due to their reduced impacts on generation costs and electricity prices, and their potential to increase the incentive for investment in in-state generation capacity (Bushnell, Holland, Hughes, and Knittel 2014). ${ }^{33}$ Given the likelihood of wide adoption of rate-based compliance approaches, it is crucial to understand the welfare implications of these alternative policies and how they will impact decision-making regarding consumption of energy efficiency and generation.

We have shown that crediting of electricity savings resulting from energy efficiency measures can yield first-best outcomes when demand for energy services is perfectly inelastic. Furthermore, we show that under inelastic demand conditions, if energy efficiency (or more accurately the savings resulting from efficiency measures) is perfectly substitutable with elec-

32 States are also permitted to use a "state-measures" approach under which the state implements a suite of indirect emissions policies, such as energy efficiency resource standards, renewable portfolio standards, planned retirements of fossil capacity and addition of low or zero-carbon generation capacity, which in aggregate achieve emissions reductions sufficient to meet the CPP targets.

${ }^{33}$ Note that these same outcomes can be achieved through a mass-based policy if emissions allowances are freely allocated based on production (Palmer and Paul 2015) 
tricity generation for producing energy services (i.e. $1 \mathrm{MWh}$ saved yields the same amount of energy services as $1 \mathrm{MWh}$ generated) then one-to-one crediting of efficiency savings achieves the first best outcome. This one-to-one crediting of electricity savings and generation is precisely the approach allowed under the EPA's CPP. Under the rate-based option for compliance with the CPP, savings resulting from energy efficiency measures create "emissions reduction credits (ERCs)" which can be added to the generation in the denominator of the intensity fraction (equivalent to equation 23). This approach effectively treats electricity savings as a form of zero-emissions generation, and thereby eliminates the distortion between generation and efficiency measures, yielding an allocation equivalent to that under an emissions tax. Furthermore, such a modification does not require an additional instrument or optimal tuning of emissions ratings to achieve first-best (Holland, Hughes, and Knittel 2009; Holland 2012; Lemoine 2013), which may make it easier to implement in practice.

Importantly, however, the optimality of the emissions standard with crediting of efficiency savings breaks down when demand for energy services exhibits some elasticity. Crediting savings from efficiency measures can no longer recover first-best, and even if crediting improves the economic efficiency of the policy (relative to providing no credit for energy efficiency measures), adjustments to the crediting rate for efficiency savings may be required to maximize welfare. Thus, under elastic demand conditions, while crediting of efficiency savings extends the output subsidy to efficiency measures thereby eliminating the relative distortion between efficiency and generation, the output subsidy still exists, except it is now a subsidy for both generation and electricity savings. Thus, the output subsidy has effectively been replaced with a subsidy for energy services. This leads to excessive consumption of energy services (both generation and energy efficiency) relative to first-best. 
Given that demand for energy services in the real world likely exhibits some elasticity, the simplified approach suggested by EPA may not achieve the optimal outcomes. However, our numerical analysis demonstrates that under most conditions, crediting of efficiency measures under an emissions intensity standard reduces costs and increases welfare relative to a standard that does not credit efficiency. Furthermore, although the optimal crediting rate for efficiency will ultimately depend on a range of factors, including the substitutability of electricity savings and electricity generation, the elasticity of demand for energy services, and the level of emissions reduction required by the policy, crediting at a rate of 1 (i.e. one-for-one crediting) may be in many relevant situations preferred over no crediting at all. ${ }^{34}$

The equivalence of crediting energy efficiency or defining the standard in terms of baseline output holds under certainty. However, in reality, determining energy efficiency activities to be credited or what baseline output should be set at may be very uncertain. Further research into whether this equivalency holds under various considerations of uncertainty may provide further useful guidance for policymakers.

\section{References}

Allcott, H. and M. Greenstone (2012). Is there an energy efficiency gap? The Journal of Economic Perspectives, 3-28.

Arimura, T. H., S. Li, R. G. Newell, and K. Palmer (2012). Cost-effectiveness of electricity energy efficiency programs. The Energy Journal 33(2), 63-99.

34 This also assumes that energy efficiency can be accurately measured, a point that has been called into question (e.g. Fowlie, Greenstone, and Wolfram (2015)). 
Bushnell, J. B., S. P. Holland, J. E. Hughes, and C. R. Knittel (2014). Strategic policy choice in state-level regulation: The EPAs Clean Power Plan. University of California Energy Institute Working Paper 255.

Fabrizio, K. R., N. L. Rose, and C. D. Wolfram (2007). Do markets reduce costs? Assessing the impact of regulatory restructuring on US electric generation efficiency. American Economic Review 97(4), 1250-1277.

Fell, H. and J. Linn (2013). Renewable electricity policies, heterogeneity, and cost effectiveness. Journal of Environmental Economics and Management 66(3), 688-707.

Fowlie, M., M. Greenstone, and C. Wolfram (2015). Do energy efficiency investments deliver? evidence from the weatherization assistance program. NBER Working Paper No. 21331.

Fullerton, D. and G. Heutel (2010). The general equilibrium incidence of environmental mandates. American Economic Journal: Economic Policy 2(3), 64-89.

Helfand, G. E. (1991). Standards versus standards: The effects of different pollution restrictions. American Economic Review 81(3), 622-634.

Holland, S. P. (2012). Emissions taxes versus intensity standards: Second-best environmental policies with incomplete regulation. Journal of Environmental Economics and Management 63(3), 375-387.

Holland, S. P., J. E. Hughes, and C. R. Knittel (2009). Greenhouse gas reductions under Low Carbon Fuel Standards? American Economic Journal: Economic Policy 1(1), $106-146$. 
Ito, K. (2014). Do consumers respond to marginal or average price? Evidence from nonlinear electricity pricing. American Economic Review 104(2), 537-563.

Lemoine, D. (2013). Escape from third-best: Rating emissions for intensity standards. University of Arizona Working Paper 12-03.

Palmer, K. and A. Paul (2015). A primer on comprehensive policy options for states to comply with the Clean Power Plan. RFF Working Paper DP 15-15. 
Table 1: Baseline Parameter Values

\begin{tabular}{|c|c|c|}
\hline Parameter & Value & Description \\
\hline$\alpha$ & 0.4 & $\begin{array}{l}\text { Productivity of energy efficiency in the pro- } \\
\text { duction of energy services }\end{array}$ \\
\hline$\sigma$ & 2 & $\begin{array}{l}\text { Elasticity of substitution between electricity } \\
\text { and energy efficiency }\end{array}$ \\
\hline$\epsilon$ & -0.1 & Energy services demand elasticity \\
\hline$c_{\theta}$ & $\$ 50 / \mathrm{MWh}$ & Marginal cost of energy efficiency \\
\hline$e_{i}^{N G}$ & 116.9lbs/MMBtu & $\mathrm{CO}_{2}$ Emissions intensity for natural gas \\
\hline$e_{i}^{C}$ & 210.6lbs/MMBtu & $\mathrm{CO}_{2}$ Emissions intensity for coal \\
\hline$P_{2020}^{N G}$ & $\$ 5.17 / \mathrm{MMBtu}$ & Assumed price of natural gas in 2020 \\
\hline$P_{2020}^{C}$ & $\$ 2.22 / \mathrm{MMBtu}$ & Assumed price of coal in 2020 \\
\hline reduction & $20 \%$ & $\begin{array}{l}\text { Target emission levels are at this percent be- } \\
\text { low } 2020 \text { baseline emissions }\end{array}$ \\
\hline
\end{tabular}

Table 2: Policy Analysis Results

\begin{tabular}{|l|l|l|l|l|l|l|l|}
\hline$\kappa$ & 0 & 0.2 & 0.4 & 0.6 & 0.8 & 1.0 & 1.2 \\
\hline Cost Ratio & 1.412 & 1.265 & 1.160 & 1.122 & 1.083 & 1.089 & 1.116 \\
\hline$(C / A)^{T P S}$ & 15.59 & 16.78 & 16.78 & 16.78 & 16.78 & 16.78 & 16.78 \\
\hline$\mu$ & 25.65 & 24.00 & 22.86 & 21.65 & 20.66 & 19.56 & 18.50 \\
\hline$I$ & 1001 & 1010 & 1013 & 1008 & 997 & 980 & 958 \\
\hline$E S^{T P S}$ & 190.5 & 191.0 & 191.4 & 191.8 & 192.2 & 192.5 & 192.7 \\
\hline$Q^{T P S}$ & 274.7 & 270.4 & 265.9 & 261.8 & 257.5 & 254.0 & 251.1 \\
\hline$\theta^{T P S}$ & 102.5 & 106.8 & 111.4 & 116.0 & 120.6 & 124.5 & 127.8 \\
\hline Avg $P_{E}^{T P S}$ & 42.31 & 41.57 & 40.98 & 40.51 & 40.15 & 39.87 & 39.65 \\
\hline \multicolumn{7}{|l|}{ Tax: $(C / A)^{T a x}=11.04, \tau=18.31, E S=188.6, Q=250.8, \theta=119.9, \mathrm{Avg} P_{E}=48.75$} \\
\hline \multicolumn{6}{|l|}{ Baseline : $: S=191.2, Q=283.4, \theta=97.8, \mathrm{Avg} P_{E}=40.15, \mathrm{CO}_{2}=171.9$} \\
\hline
\end{tabular}

Notes: "Tax" and "Baseline" values are for the tax policy and baseline case with no policy, respectively, and are constant across all $\kappa$ values. Remaining values are for the TPS policy. "Cost Ratio" gives the ratio of $(C / A)^{T P S}$ to $(C / A)^{T a x}$. $\mu$ and $\tau$ are in $\$ / t C O_{2}$. ES values are in millions of ES units. $Q$ and $\theta$ values are in million MWhs. Avg. $P_{E}$ values give the quantity weighted average electricity prices in $\$ / M W h$. In the "Baseline" row, "COO ${ }_{2}$ gives emissions in million $\mathrm{tCO}_{2}$ and the emissions of the policies are $20 \%$ below that value. 
Figure 1: Sensitivity Analysis - Demand Parameters
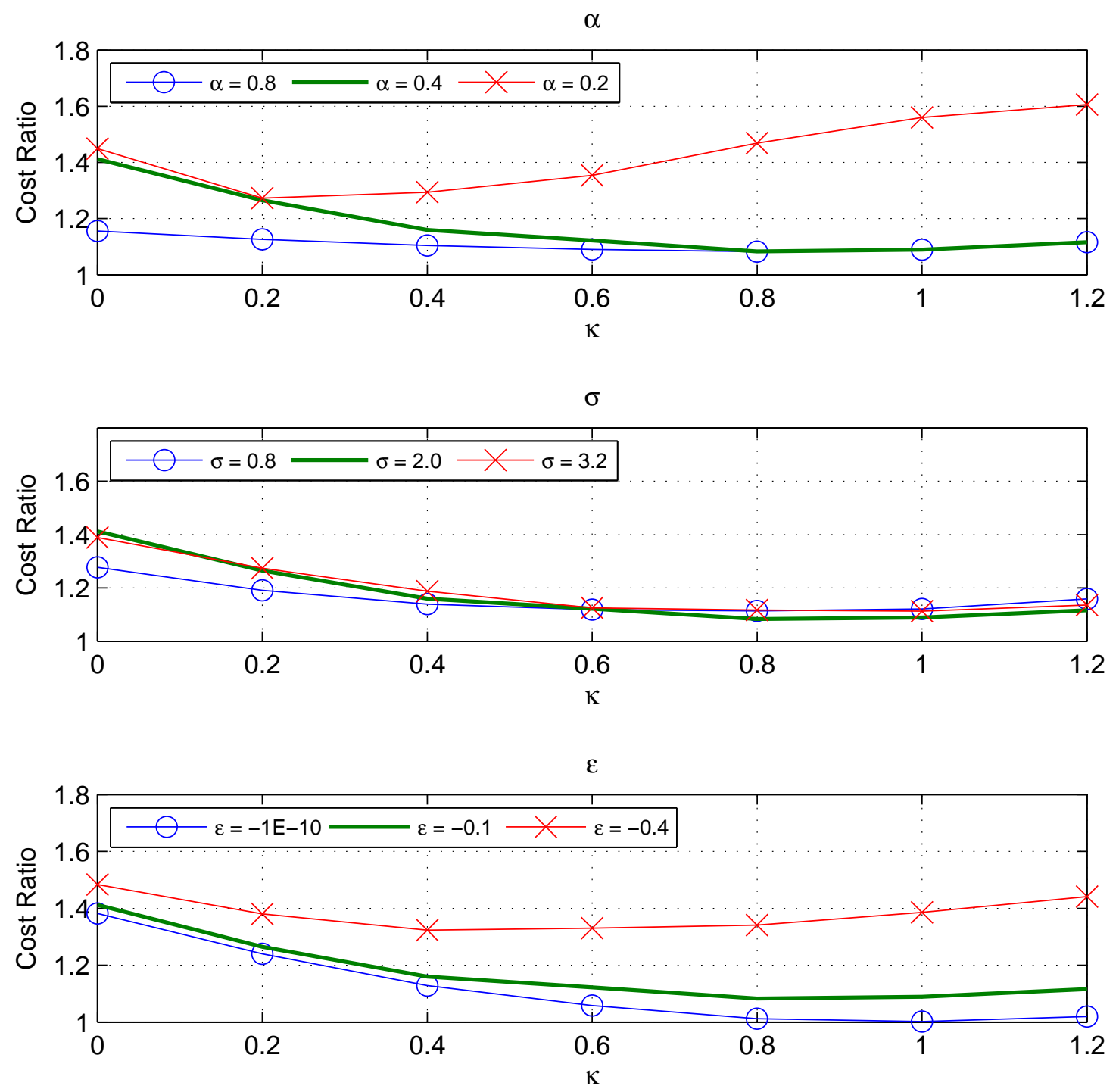
Figure 2: Sensitivity Analysis - Market and Policy Parameters
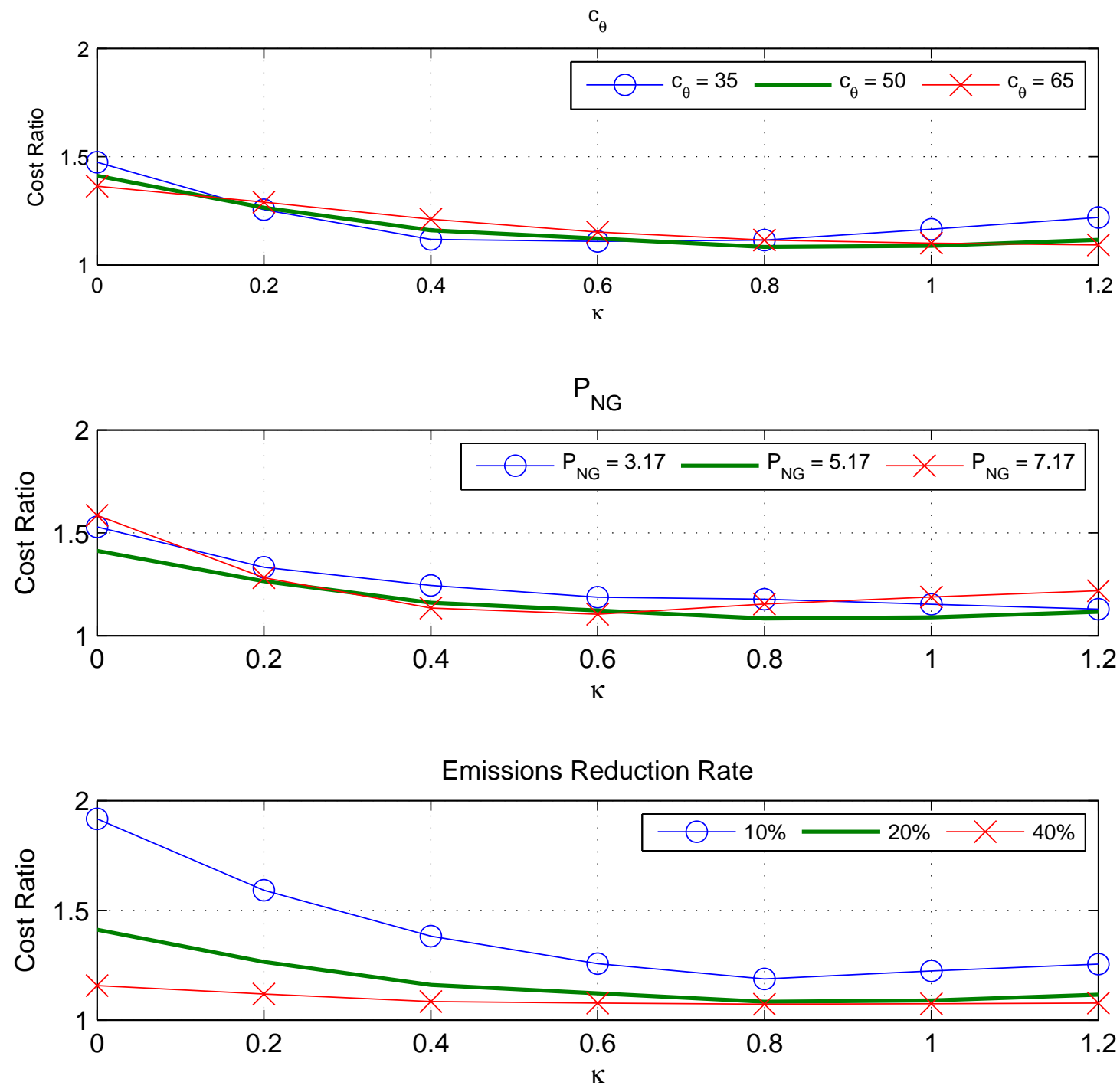


\section{A Analytical Proofs}

\section{A.1 Proof of Lemma 1}

To establish the first part of Lemma 1, taking the first-order conditions of equation 6 yields:

$$
\begin{aligned}
\frac{\partial L}{\partial Q_{i}} & =c_{i}\left(Q_{i}\right)+\tau \gamma_{i}-\lambda \frac{\partial E S}{\partial Q}=0, \quad \forall i \\
\frac{\partial L}{\partial \theta} & =\frac{d e}{d \theta}-\lambda \frac{\partial E S}{\partial \theta}=0
\end{aligned}
$$

Rearranging and solving for $\lambda$ establishes that marginal social costs of energy services are equated, as displayed in equation 7 .

To establish that energy efficiency is always greater under an emissions tax relative to the unregulated case, compare equation 7 with 5 . The emissions tax raises the marginal cost for at least some generator (since by assumption $\gamma_{i}>0$ for some generator) relative to the unregulated case. Given that the demand for energy services is fixed, the firm substitutes towards providing more energy efficiency.

\section{A.2 Proof of Proposition 1}

To establish the first part of Proposition 1, taking the first-order conditions of equation 8 and setting $f^{\prime}(\theta)=0$ yields:

$$
\begin{aligned}
\frac{\partial L}{\partial Q_{i}} & =c_{i}\left(Q_{i}\right)+\mu \gamma_{i}-\mu I-\lambda \frac{\partial E S}{\partial Q}=0, \quad \forall i \\
\frac{\partial L}{\partial \theta} & =\frac{d e}{d \theta}-\lambda \frac{\partial E S}{\partial \theta}=0 .
\end{aligned}
$$


Combining the above equations and comparing with the optimal conditions established in Lemma 1 shows that the emissions intensity standard cannot recover the first-best allocation. Optimality under the emissions intensity standard would require that $\tau \gamma_{i}=\mu\left(\gamma_{i}-I\right) \forall i$. However, if $\tau \gamma_{i}=\mu\left(\gamma_{i}-I\right)$, then $\mu=\frac{\tau \gamma_{i}}{\gamma_{i}-I}$, and thus $\tau \gamma_{j}=\frac{\tau \gamma_{i}}{\gamma_{i}-I}\left(\gamma_{i}-I\right)$. Rearranging, this requires $\frac{\gamma_{i}}{\gamma_{j}}=\frac{\gamma_{i}-I}{\gamma_{j}-I}$, or that either $\gamma_{i}=\gamma_{j} \forall i, j$ or $I=0$, both of which have been ruled out by assumption.

To show the equivalence of the allocation under an emissions intensity standard with that under an emissions tax coupled with an energy efficiency tax, let $\delta$ represent a tax on energy efficiency $\theta$. Then the firm's problem is to solve:

$$
L=\sum_{i}^{N} \int_{0}^{Q_{i}} c_{i}\left(q_{i}\right) d q_{i}+e(\theta)+\tau\left(\sum_{i}^{N} \gamma_{i} Q_{i}\right)+\delta \theta+\lambda(\overline{E S}-E S(Q, \theta))
$$

with first-order conditions:

$$
\begin{aligned}
\frac{\partial L}{\partial Q_{i}} & =c_{i}\left(Q_{i}\right)+\tau \gamma_{i}-\lambda \frac{\partial E S}{\partial Q}=0, \quad \forall i \\
\frac{\partial L}{\partial \theta} & =\frac{d e}{d \theta}+\delta-\lambda \frac{\partial E S}{\partial \theta}=0
\end{aligned}
$$

Rearranging and comparing with the emissions intensity standard conditions, an energy efficiency tax of $\delta=\mu I \frac{\partial E S / \partial \theta}{\partial E S / \partial Q}$ would yield an identical allocation. 


\section{A.3 Proof of Proposition 2}

The first-order conditions of equation 8 are as follows:

$$
\begin{aligned}
\frac{\partial L}{\partial Q_{i}} & =c_{i}\left(Q_{i}\right)+\mu \gamma_{i}-\mu I-\lambda \frac{\partial E S}{\partial Q}=0, \quad \forall i \\
\frac{\partial L}{\partial \theta} & =\frac{d e}{d \theta}-\mu I f^{\prime}(\theta)-\lambda \frac{\partial E S}{\partial \theta}=0 .
\end{aligned}
$$

Comparing condition $\frac{\partial L}{\partial \theta}$ in the above to that in equation 26 shows that crediting introduces an energy efficiency subsidy equal to $\mu I f^{\prime}(\theta)$. If $f^{\prime}(\theta)$ is set equal to the ratio of energy service production $\frac{\partial E S / \partial \theta}{\partial E S / \partial Q}$, then the $\mu I$ terms disappear from equation 10, and if the standard is set such that $\mu=\tau$, then all terms are equivalent to the first-best solution in equation 7 .

\section{A.4 Proof of Lemma 2}

First-order conditions from the household problem in equation 12 yield:

$$
\begin{aligned}
& P(E S(Q, \theta)) \frac{\partial E S}{\partial Q}=P_{E} \\
& P(E S(Q, \theta)) \frac{\partial E S}{\partial \theta}=P_{\theta} .
\end{aligned}
$$

Households equate the marginal benefit of additional energy consumption and energy efficiency to their respective prices. 
First-order conditions for the firm in equation 13 are:

$$
\begin{aligned}
P_{E} & =c_{i}\left(Q_{i}\right)+\tau \gamma_{i} \quad \forall i \\
P_{\theta} & =\frac{d e}{d \theta} .
\end{aligned}
$$

Combining the above first-order conditions for the household and the firm yields the conditions in Lemma 2.

\section{A.5 Proof of Proposition 3}

Taking the first order conditions for the firm's problem in equation 15 and setting $f^{\prime}(\theta)=0$ :

$$
\begin{aligned}
P_{E} & =c_{i}\left(Q_{i}\right)+\tau \gamma_{i}+\mu \gamma_{i}-\mu I \quad \forall i \\
P_{\theta} & =\frac{d e}{d \theta} .
\end{aligned}
$$

Combining the first-order conditions for the representative household and firm yields:

$$
\begin{aligned}
& P(E S(Q, \theta)) \frac{\partial E S}{\partial Q}=c_{i}\left(Q_{i}\right)+\mu \gamma_{i}-\mu I \quad \forall i \\
& P(E S(Q, \theta)) \frac{\partial E S}{\partial \theta}=\frac{d e}{d \theta} .
\end{aligned}
$$

Comparing these conditions with those in Lemma 2 shows the traditional emissions intensity standard cannot recover the first-best allocation. Furthermore, when the standard is set such that $\mu=\tau$, the equilibrium price of energy services $P(E S(Q, \theta)$ is less than that under first-best, leading to too little energy efficiency under the intensity standard. 


\section{A.6 Proof of Proposition 4}

Taking the first order conditions for the firm's problem in equation 15:

$$
\begin{aligned}
& P_{E}=c_{i}\left(Q_{i}\right)+\tau \gamma_{i}+\mu \gamma_{i}-\mu I \quad \forall i \\
& P_{\theta}=\frac{d e}{d \theta}-\mu I f^{\prime}(\theta)
\end{aligned}
$$

Combining the first-order conditions for the representative household and firm yields:

$$
\begin{aligned}
& P(E S(Q, \theta)) \frac{\partial E S}{\partial Q}=c_{i}\left(Q_{i}\right)+\mu \gamma_{i}-\mu I \quad \forall i \\
& P(E S(Q, \theta)) \frac{\partial E S}{\partial \theta}=\frac{d e}{d \theta}-\mu I f^{\prime}(\theta)
\end{aligned}
$$

Comparing these conditions with those in Lemma 2, the presence of the implicit subsidy terms coupled with downward-sloping inverse demand, $P(E S(Q, \theta))$, shows that the emissions intensity standard cannot recover the first-best allocation with elastic demand for energy services.

To show the second part of Proposition 4, rearranging equations 14 and 37 to express terms in dollars per unit of energy service shows that crediting at $f^{\prime}(\theta)=\frac{\partial E S / \partial \theta}{\partial E S / \partial Q}$ offsets the relative distortion favoring the generation sector. 


\section{A.7 Proof of Proposition 5}

The optimal $\kappa$ satisfies:

$$
\frac{d W}{d \kappa}=\left(P(E S) \frac{\partial E S}{\partial \theta}-\frac{d e}{d \theta}\right) \frac{d \theta}{d \kappa}+\sum_{i}^{N}\left[\left(P(E S) \frac{\partial E S}{\partial Q}-c_{i}-\tau \gamma_{i}\right) \frac{d Q_{i}}{d \kappa}\right]=0
$$

The change in welfare due to a change in the crediting rule is equal to the wedge between marginal benefits and costs in the efficiency sector times the change in efficiency, plus the wedge between marginal benefits and social costs in all of the electricity generating sectors times the change in respective generation. Equation 39 can thus be interpreted as a series of marginal deadweight losses associated with changes in $\kappa \cdot{ }^{35}$ Plugging in the first-order conditions from the emissions intensity standard with crediting (equation 37) yields equation 17.

\section{A.8 Proof of Proposition 6}

First, differentiate the energy services production function $\operatorname{ES}(Q(\kappa), \theta(\kappa))$ to obtain:

$$
\frac{d E S}{d \kappa}=\frac{\partial E S}{\partial Q} \sum_{i}^{N} \frac{d Q_{i}}{d \kappa}+\frac{\partial E S}{\partial \theta} \frac{d \theta}{d \kappa}
$$

Rearranging equation 40 to solve for $\frac{d e}{d \kappa}$ and substituting into equation 17 yields:

$$
\frac{d W}{d \kappa}=(-\mu I \kappa) \frac{d E S / d \kappa}{\partial E S / \partial \theta}+\sum_{i}^{N}\left[\left(\mu I \kappa \frac{\partial E S / \partial Q}{\partial E S / \partial \theta}+\mu\left(\gamma_{i}-I\right)-\tau \gamma_{i}\right) \frac{d Q_{i}}{d \kappa}\right]=0
$$

${ }^{35}$ Note that the terms in parenthesis are equivalent to the first-order conditions from the emission tax (equation 14). 
The first term reflects the distortion created by the elastic demand response to the crediting rule $(d E S / d \kappa)$, while the second term reflects the distortions associated with the generation sector.

With inelastic demand, $d E S / d \kappa=0$, so the first term simply drops from equation 41 . Suppose the crediting rule is set such that $\kappa=\frac{\partial E S / \partial \theta}{\partial E S / \partial Q}$. Then equation 41 simply becomes $\sum_{i}^{N}\left[(\mu-\tau) \gamma_{i} \frac{d Q_{i}}{d \kappa}\right]=0$, which is satisfied provided the standard is set such that $\mu=\tau$. Alternatively, suppose the regulator sets the standard such that $\mu=\tau$. Then equation 41 requires an $\kappa$ such that $\left.\sum_{i}^{N}\left[\tau I \kappa \frac{\partial E S / \partial Q}{\partial E S / \partial \theta}-\tau I\right) \frac{d Q_{i}}{d \kappa}\right]=0$. Rearranging and solving yields the optimal rule $\kappa=\frac{\partial E S / \partial \theta}{\partial E S / \partial Q}$.

To show that no other combination of $\kappa$ and $\mu$ can satisfy equation 41 , suppose that the standard is set arbitrarily. Then satisfying equation 41 requires setting an $\kappa$ such that $\left(\mu I \kappa \frac{\partial E S / \partial Q}{\partial E S / \partial \theta}+(\mu-\tau) \gamma_{i}-\mu I\right)=0 \quad \forall i$, which is impossible (given that $\gamma_{i} \neq \gamma_{j}$ for some $i, j$ ). Similarly, by setting $\kappa$ arbitrarily, it is impossible to find a $\mu$ that satisfies equation 41 .

\section{A.9 Proof of Proposition 7}

With elastic demand, the first term in equation 41 is always negative due to $d E S / d \kappa>0$. Again, consider the crediting rule $\kappa=\frac{\partial E S / \partial \theta}{\partial E S / \partial Q}$. Then equation 41 becomes $\left(-\mu I \frac{d E S / d \kappa}{\partial E S / \partial Q}\right)+$ $(\mu-\tau) \sum_{i}^{N}\left[\gamma_{i} \frac{d Q_{i}}{d \kappa}\right]=0$. Setting the standard such that $\mu=\tau$ is clearly not optimal due to the presence of the (negative) first term arising from the absolute distortion from the subsidy. If emissions fall as crediting rises $\left(\sum_{i}^{N}\left[\gamma_{i} \frac{d Q_{i}}{d \kappa}\right]<0\right)$, then for equation 41 to hold, $\mu<\tau$, and conversely if emissions increase. Next, if the regulator sets the standard such that $\mu=\tau$, then equation 41 requires $-\kappa \frac{d E S / d \kappa}{\partial E S / \partial \theta}+\left(\kappa \frac{\partial E S / \partial Q}{\partial E S / \partial \theta}-1\right) \sum_{i}^{N}\left[\frac{d Q_{i}}{d \kappa}\right]=0$. Setting 
$\kappa=\frac{\partial E S / \partial \theta}{\partial E S / \partial Q}$ is again not optimal due to the presence of the negative first term. If generation falls as the crediting rule increases, $\sum_{i}^{N}\left[\frac{d Q_{i}}{d \kappa}\right]<0$, then the optimal crediting rule is such that $\kappa<\frac{\partial E S / \partial \theta}{\partial E S / \partial Q}$, and conversely $\kappa>\frac{\partial E S / \partial \theta}{\partial E S / \partial Q}$ if generation rises. Finally, setting $\mu=\tau$ in equation 41 and rearranging and implicitly solving for $\kappa$ yields equation 18.

\section{B Sensitivity Analysis Results Summary}

Below are the tables giving more details on the outputs from the sensitivity analyses. At the top of each table is the variable that was altered in the analysis. All other variables not listed otherwise are those given in Table 1 . Note also that Figures 1 and 2 contain three plots for each parameter sensitivity analysis. The middle parameter value in each of these analyses is simply the reference case results. A summary of the outputs from the reference case is given in Table 2 . 
Table B.1: $\alpha$ Sensitivity

$\alpha=0.8$

\begin{tabular}{|l|l|l|l|l|l|l|l|}
\hline$\kappa$ & 0 & 0.2 & 0.4 & 0.6 & 0.8 & 1.0 & 1.2 \\
\hline Cost Ratio & 1.449 & 1.273 & 1.294 & 1.354 & 1.469 & 1.560 & 1.606 \\
\hline$(C / A)^{T P S}$ & 6.40 & 5.63 & 5.72 & 5.98 & 6.49 & 6.89 & 7.10 \\
\hline$\mu$ & 18.58 & 16.32 & 14.36 & 12.62 & 11.33 & 10.33 & 9.54 \\
\hline$I$ & 1060 & 1055 & 1027 & 987 & 944 & 902 & 862 \\
\hline$E S^{T P S}$ & 2201.5 & 2209.1 & 2214.6 & 2218.3 & 2220.9 & 2222.7 & 2224.0 \\
\hline$Q^{T P S}$ & 237.7 & 233.5 & 230.4 & 228.5 & 227.4 & 226.6 & 226.0 \\
\hline$\theta^{T P S}$ & 3048.7 & 3063.3 & 3074.0 & 3081.1 & 3085.9 & 3089.4 & 3091.9 \\
\hline Avg $P_{E}^{T P S}$ & 39.46 & 38.54 & 37.87 & 37.42 & 37.09 & 36.86 & 36.68 \\
\hline Tax: $(C / A)^{T a x}=4.42, \tau=8.87, E S=2201.1, Q=226.4, \theta=3057.7, \mathrm{Avg} P_{E}=40.46$ \\
\hline Baseline : $E S=2202.0, Q=259.6, \theta=3036.7, \mathrm{Avg} P_{E}=37.25, \mathrm{CO}_{2}=157.5$ \\
\hline
\end{tabular}

$$
\alpha=0.2
$$

\begin{tabular}{|l|l|l|l|l|l|l|l|}
\hline$\kappa$ & 0 & 0.2 & 0.4 & 0.6 & 0.8 & 1.0 & 1.2 \\
\hline Cost Ratio & 1.156 & 1.126 & 1.104 & 1.090 & 1.082 & 1.089 & 1.116 \\
\hline$(C / A)^{T P S}$ & 20.09 & 19.57 & 19.19 & 18.94 & 18.80 & 18.92 & 19.40 \\
\hline$\mu$ & 30.64 & 29.81 & 28.95 & 28.39 & 27.59 & 26.77 & 26.06 \\
\hline$I$ & 971 & 973 & 974 & 972 & 968 & 961 & 950 \\
\hline$E S^{T P S}$ & 213.6 & 213.8 & 214.1 & 214.3 & 214.5 & 214.6 & 214.8 \\
\hline$Q^{T P S}$ & 298.2 & 296.8 & 295.4 & 293.8 & 292.1 & 290.4 & 288.7 \\
\hline$\theta^{T P S}$ & 15.8 & 17.4 & 19.0 & 21.0 & 23.0 & 25.2 & 27.6 \\
\hline Avg $P_{E}^{T P S}$ & 44.11 & 43.74 & 43.37 & 43.09 & 42.79 & 42.53 & 42.34 \\
\hline $\operatorname{Tax}:(C / A)$ &
\end{tabular}

Tax: $(C / A)^{\operatorname{Tax}}=17.38, \tau=25.07, E S=209.4, Q=285.3, \theta=22.1, \operatorname{Avg} P_{E}=54.20$

Baseline : $E S=215.0, Q=302.0, \theta=14.4, \operatorname{Avg} P_{E}=41.37, \mathrm{CO}_{2}=180.9$

Notes: "Tax" and "Baseline" values are for the tax policy and baseline case with no policy, respectively, and are constant across all $\kappa$ values. Remaining values are for the TPS policy. "Cost Ratio" gives the ratio of $(C / A)^{T P S}$ to $(C / A)^{T a x}$. $\mu$ and $\tau$ are in $\$ / t C O_{2}$. ES values are in millions of ES units. $Q$ and $\theta$ values are in million $M W h s$. Avg. $P_{E}$ values give the quantity weighted average electricity prices in $\$ / M W h$. In the "Baseline" row, "COO emissions in million $\mathrm{tCO}_{2}$ and the emissions of the policies are $20 \%$ below that value. 
Table B.2: $\sigma$ Sensitivity

$\sigma=0.8$

\begin{tabular}{|l|l|l|l|l|l|l|l|}
\hline$\kappa$ & 0 & 0.2 & 0.4 & 0.6 & 0.8 & 1.0 & 1.2 \\
\hline Cost Ratio & 1.277 & 1.191 & 1.139 & 1.119 & 1.114 & 1.121 & 1.159 \\
\hline$(C / A)^{T P S}$ & 16.78 & 15.65 & 14.97 & 14.71 & 14.64 & 14.73 & 15.23 \\
\hline$\mu$ & 26.90 & 25.73 & 24.52 & 23.60 & 22.84 & 22.02 & 21.33 \\
\hline$I$ & 991 & 997 & 996 & 991 & 980 & 964 & 943 \\
\hline$E S^{T P S}$ & 231.6 & 232.3 & 233.0 & 233.5 & 234.1 & 234.5 & 234.9 \\
\hline$Q^{T P S}$ & 280.4 & 277.2 & 274.3 & 271.2 & 268.2 & 265.3 & 262.7 \\
\hline$\theta^{T P S}$ & 177.0 & 180.8 & 184.6 & 188.5 & 192.5 & 196.2 & 199.7 \\
\hline Avg $P_{E}^{T P S}$ & 42.15 & 41.61 & 41.14 & 40.81 & 40.56 & 40.37 & 40.25 \\
\hline Tax: $(C / A)^{T a x}=13.14, \tau=21.01, E S=229.3, Q=261.2, \theta=189.8, \mathrm{Avg} P_{E}=50.15$ \\
\hline
\end{tabular}

$$
\sigma=3.2
$$

\begin{tabular}{|l|l|l|l|l|l|l|l|}
\hline$\kappa$ & 0 & 0.2 & 0.4 & 0.6 & 0.8 & 1.0 & 1.2 \\
\hline Cost Ratio & 1.389 & 1.274 & 1.188 & 1.125 & 1.117 & 1.113 & 1.136 \\
\hline$(C / A)^{T P S}$ & 17.10 & 15.68 & 14.62 & 13.84 & 13.75 & 13.70 & 13.99 \\
\hline$\mu$ & 26.74 & 25.21 & 23.75 & 22.58 & 21.37 & 20.36 & 19.30 \\
\hline$I$ & 992 & 1001 & 1004 & 1000 & 988 & 970 & 947 \\
\hline$E S^{T P S}$ & 201.3 & 201.9 & 202.4 & 202.8 & 203.2 & 203.5 & 203.7 \\
\hline$Q^{T P S}$ & 282.5 & 278.4 & 274.0 & 269.2 & 264.9 & 260.4 & 256.7 \\
\hline$\theta^{T P S}$ & 140.4 & 145.0 & 149.9 & 155.1 & 160.1 & 165.2 & 169.4 \\
\hline Avg $P_{E}^{T P S}$ & 43.13 & 42.35 & 41.69 & 41.19 & 40.80 & 40.52 & 40.33 \\
\hline $\operatorname{Tax}:(C / A)$ &
\end{tabular}

Tax: $(C / A)^{\text {Tax }}=12.31, \tau=19.28, E S=199.2, Q=256.8, \theta=159.3, \operatorname{Avg} P_{E}=50.01$

Baseline : $E S=202.1, Q=289.5, \theta=136.5, \operatorname{Avg} P_{E}=41.09, \mathrm{CO}_{2}=175.2$

Notes: "Tax" and "Baseline" values are for the tax policy and baseline case with no policy, respectively, and are constant across all $\kappa$ values. Remaining values are for the TPS policy. "Cost Ratio" gives the ratio of $(C / A)^{T P S}$ to $(C / A)^{T a x}$. $\mu$ and $\tau$ are in $\$ / t C O_{2}$. ES values are in millions of ES units. $Q$ and $\theta$ values are in million $M W h s$. Avg. $P_{E}$ values give the quantity weighted average electricity prices in $\$ / M W h$. In the "Baseline" row, "COO emissions in million $\mathrm{tCO}_{2}$ and the emissions of the policies are $20 \%$ below that value. 
Table B.3: $\epsilon$ Sensitivity $\epsilon=-1.0 \mathrm{E}^{-10}$

\begin{tabular}{|l|l|l|l|l|l|l|l|}
\hline$\kappa$ & 0 & 0.2 & 0.4 & 0.6 & 0.8 & 1.0 & 1.2 \\
\hline Cost Ratio & 1.382 & 1.240 & 1.128 & 1.058 & 1.012 & 1.002 & 1.020 \\
\hline$(C / A)^{T P S}$ & 16.15 & 14.49 & 13.18 & 12.37 & 11.83 & 11.71 & 11.92 \\
\hline$\mu$ & 26.94 & 25.03 & 23.45 & 22.09 & 20.95 & 19.81 & 18.69 \\
\hline$I$ & 993 & 1003 & 1007 & 1005 & 996 & 981 & 961 \\
\hline$E S^{T P S}$ & 194.8 & 194.8 & 194.8 & 194.8 & 194.8 & 194.8 & 194.8 \\
\hline$Q^{T P S}$ & 279.1 & 274.4 & 269.6 & 264.9 & 260.4 & 256.6 & 253.5 \\
\hline$\theta^{T P S}$ & 107.2 & 111.1 & 115.2 & 119.3 & 123.4 & 127.1 & 130.2 \\
\hline Avg $P_{E}^{T P S}$ & 42.69 & 41.82 & 41.12 & 40.57 & 40.12 & 39.78 & 39.52 \\
\hline Tax: $(C / A)^{T a x}=11.69, \tau=19.57, E S=194.8, Q=255.9, \theta=127.6, \mathrm{Avg} P_{E}=49.60$ \\
\hline Baseline : $E S=173.6, Q=267.7, \theta=76.0, \mathrm{Avg} P_{E}=37.49, \mathrm{CO}_{2}=173.2$ \\
\hline
\end{tabular}

$$
\epsilon=-0.4
$$

\begin{tabular}{|l|l|l|l|l|l|l|l|}
\hline$\kappa$ & 0 & 0.2 & 0.4 & 0.6 & 0.8 & 1.0 & 1.2 \\
\hline Cost Ratio & 1.484 & 1.380 & 1.323 & 1.330 & 1.341 & 1.386 & 1.441 \\
\hline$(C / A)^{T P S}$ & 12.94 & 12.04 & 11.53 & 11.60 & 11.69 & 12.08 & 12.57 \\
\hline$\mu$ & 23.75 & 22.83 & 21.82 & 21.00 & 20.03 & 19.04 & 18.16 \\
\hline$I$ & 1018 & 1023 & 1020 & 1008 & 989 & 965 & 937 \\
\hline$E S^{T P S}$ & 181.0 & 182.6 & 184.1 & 185.4 & 186.5 & 187.4 & 188.2 \\
\hline$Q^{T P S}$ & 263.3 & 260.3 & 257.1 & 253.9 & 250.9 & 248.2 & 245.8 \\
\hline$\theta^{T P S}$ & 96.7 & 102.1 & 107.5 & 113.1 & 118.2 & 122.6 & 126.5 \\
\hline Avg $P_{E}^{T P S}$ & 41.54 & 41.06 & 40.65 & 40.35 & 40.10 & 39.92 & 39.78 \\
\hline
\end{tabular}

Tax: $(C / A)^{T a x}=8.72, \tau=17.72, E S=175.5, Q=240.1, \theta=106.9, \operatorname{Avg} P_{E}=46.34$

Baseline : $E S=184.0, Q=275.5, \theta=93.0, \operatorname{Avg} P_{E}=39.27, \mathrm{CO}_{2}=167.6$

Notes: "Tax" and "Baseline" values are for the tax policy and baseline case with no policy, respectively, and are constant across all $\kappa$ values. Remaining values are for the TPS policy. "Cost Ratio" gives the ratio of $(C / A)^{T P S}$ to $(C / A)^{\text {Tax }}$. $\mu$ and $\tau$ are in $\$ / t C O_{2}$. ES values are in millions of ES units. $Q$ and $\theta$ values are in million MWhs. Avg. $P_{E}$ values give the quantity weighted average electricity prices in $\$ / M W h$. In the "Baseline" row, "CO 2 " gives emissions in million $\mathrm{tCO}_{2}$ and the emissions of the policies are $20 \%$ below that value. 
Table B.4: $c_{\theta}$ Sensitivity

$c_{\theta}=\$ 35 / \mathrm{MWh}$ avoided

\begin{tabular}{|l|l|l|l|l|l|l|l|}
\hline$\kappa$ & 0 & 0.2 & 0.4 & 0.6 & 0.8 & 1.0 & 1.2 \\
\hline Cost Ratio & 1.474 & 1.255 & 1.118 & 1.109 & 1.115 & 1.165 & 1.220 \\
\hline$(C / A)^{T P S}$ & 14.04 & 11.95 & 10.65 & 10.56 & 10.62 & 11.10 & 11.62 \\
\hline$\mu$ & 24.41 & 22.43 & 20.61 & 18.71 & 17.10 & 15.90 & 14.83 \\
\hline$I$ & 1011 & 1023 & 1020 & 999 & 968 & 931 & 893 \\
\hline$E S^{T P S}$ & 232.8 & 233.7 & 234.6 & 235.2 & 235.6 & 236.0 & 236.3 \\
\hline$Q^{T P S}$ & 268.3 & 261.4 & 254.6 & 249.3 & 245.1 & 241.8 & 239.4 \\
\hline$\theta^{T P S}$ & 203.2 & 213.4 & 223.6 & 232.1 & 238.9 & 244.5 & 248.6 \\
\hline Avg $P_{E}^{T P S}$ & 41.89 & 40.86 & 40.09 & 39.54 & 39.14 & 38.84 & 38.63 \\
\hline \multicolumn{7}{|l|}{ Tax: $(C / A)^{T a x}=9.52, \tau=16.64, E S=231.0, Q=244.0, \theta=228.8}$, & $\mathrm{Avg} P_{E}=47.33$ \\
\hline Baseline : $E S=233.6, Q=278.8, \theta=195.0, \operatorname{Avg} P_{E}=39.72, \mathrm{CO}_{2}=169.5$ \\
\hline
\end{tabular}

$c_{\theta}=\$ 65 / \mathrm{MWh}$ avoided

\begin{tabular}{|l|l|l|l|l|l|l|l|}
\hline$\kappa$ & 0 & 0.2 & 0.4 & 0.6 & 0.8 & 1.0 & 1.2 \\
\hline Cost Ratio & 1.364 & 1.290 & 1.211 & 1.152 & 1.115 & 1.101 & 1.093 \\
\hline$(C / A)^{T P S}$ & 16.47 & 15.57 & 14.62 & 13.90 & 13.46 & 13.29 & 13.20 \\
\hline$\mu$ & 26.27 & 25.28 & 24.16 & 23.37 & 22.55 & 21.75 & 21.04 \\
\hline$I$ & 995 & 1001 & 1005 & 1005 & 1001 & 994 & 983 \\
\hline$E S^{T P S}$ & 169.7 & 170.0 & 170.3 & 170.6 & 170.9 & 171.1 & 171.3 \\
\hline$Q^{T P S}$ & 279.2 & 276.3 & 273.3 & 270.2 & 267.1 & 264.1 & 261.2 \\
\hline$\theta^{T P S}$ & 61.9 & 64.2 & 66.6 & 69.1 & 71.6 & 74.1 & 76.6 \\
\hline Avg $P_{E}^{T P S}$ & 42.58 & 42.06 & 41.57 & 41.18 & 40.84 & 40.57 & 40.34 \\
\hline
\end{tabular}

Tax: $(C / A)^{T a x}=12.07, \tau=19.56, E S=167.7, Q=256.1, \theta=74.5, \operatorname{Avg} P_{E}=49.75$

Baseline : $E S=170.46, Q=286.8, \theta=58.7, \operatorname{Avg} P_{E}=40.42, \mathrm{CO}_{2}=175.6$

Notes: "Tax" and "Baseline" values are for the tax policy and baseline case with no policy, respectively, and are constant across all $\kappa$ values. Remaining values are for the TPS policy. "Cost Ratio" gives the ratio of (C/A) ${ }^{T} P S$ to $(C / A)^{\text {Tax }} . \mu$ and $\tau$ are in $\$ / t C \mathrm{O}_{2}$. ES values are in millions of ES units. $Q$ and $\theta$ values are in million $M$ Whs. Avg. $P_{E}$ values give the quantity weighted average electricity prices in $\$ / M W h$. In the "Baseline" row, "CO ${ }_{2}$ " gives emissions in million $\mathrm{tCO}_{2}$ and the emissions of the policies are $20 \%$ below that value. 
Table B.5: $P_{N G}$ Sensitivity

$P_{N G}=\$ 3.17 / \mathrm{MMBtu}$

\begin{tabular}{|l|l|l|l|l|l|l|l|}
\hline$\kappa$ & 0 & 0.2 & 0.4 & 0.6 & 0.8 & 1.0 & 1.2 \\
\hline Cost Ratio & 1.529 & 1.332 & 1.244 & 1.187 & 1.177 & 1.153 & 1.129 \\
\hline$(C / A)^{T P S}$ & 6.82 & 5.94 & 5.55 & 5.30 & 5.25 & 5.14 & 5.04 \\
\hline$\mu$ & 34.56 & 27.30 & 22.93 & 19.89 & 17.61 & 15.83 & 14.33 \\
\hline$I$ & 923 & 923 & 918 & 912 & 904 & 895 & 886 \\
\hline$E S^{T P S}$ & 193.8 & 194.4 & 194.8 & 195.2 & 195.4 & 195.6 & 195.8 \\
\hline$Q^{T P S}$ & 312.0 & 309.8 & 308.1 & 306.7 & 305.5 & 304.5 & 303.8 \\
\hline$\theta^{T P S}$ & 82.5 & 84.4 & 86.0 & 87.2 & 88.3 & 89.1 & 89.7 \\
\hline Avg $P_{E}^{T P S}$ & 33.87 & 32.91 & 32.28 & 31.83 & 31.51 & 31.26 & 31.06 \\
\hline Tax: $(C / A)^{T a x}=4.46, \tau=11.65, E S=192.8, Q=301.4, \theta=86.1, \mathrm{Avg} P_{E}=36.12$ \\
\hline Baseline : $E S=195.4, Q=330.4, \theta=71.8, \mathrm{Avg} P_{E}=30.38, \mathrm{CO}_{2}=180.1$ \\
\hline
\end{tabular}

\section{$P_{N G}=7.17 / \mathrm{MMBtu}$}

\begin{tabular}{|l|l|l|l|l|l|l|l|}
\hline$\kappa$ & 0 & 0.2 & 0.4 & 0.6 & 0.8 & 1.0 & 1.2 \\
\hline Cost Ratio & 1.585 & 1.282 & 1.134 & 1.104 & 1.154 & 1.188 & 1.218 \\
\hline$(C / A)^{T P S}$ & 20.48 & 16.56 & 14.65 & 14.27 & 14.91 & 15.35 & 15.74 \\
\hline$\mu$ & 41.54 & 38.00 & 34.55 & 30.55 & 27.17 & 24.41 & 22.28 \\
\hline$I$ & 1061 & 1075 & 1070 & 1047 & 1016 & 982 & 949 \\
\hline$E S^{T P S}$ & 187.9 & 188.9 & 189.7 & 190.3 & 190.8 & 191.2 & 191.5 \\
\hline$Q^{T P S}$ & 242.3 & 235.5 & 229.4 & 225.6 & 223.7 & 222.6 & 221.9 \\
\hline$\theta^{T P S}$ & 127.2 & 136.3 & 145.0 & 151.0 & 154.9 & 157.3 & 159.0 \\
\hline Avg $P_{E}^{T P S}$ & 51.25 & 49.59 & 48.20 & 47.05 & 46.16 & 45.43 & 44.87 \\
\hline
\end{tabular}

Tax: $(C / A)^{\text {Tax }}=12.92, \tau=23.86, E S=186.5, Q=223.7, \theta=145.2, \operatorname{Avg} P_{E}=57.3$

Baseline : $E S=189.2, Q=258.0, \theta=118.4, \operatorname{Avg} P_{E}=46.56, \mathrm{CO}_{2}=160.6$

Notes: "Tax" and "Baseline" values are for the tax policy and baseline case with no policy, respectively, and are constant across all $\kappa$ values. Remaining values are for the TPS policy. "Cost Ratio" gives the ratio of $(C / A)^{T P S}$ to $(C / A)^{T a x}$. $\mu$ and $\tau$ are in $\$ / t C O_{2}$. ES values are in millions of ES units. $Q$ and $\theta$ values are in million MWhs. Avg. $P_{E}$ values give the quantity weighted average electricity prices in $\$ / M W h$. In the "Baseline" row, "COO emissions in million $\mathrm{tCO}_{2}$ and the emissions of the policies are $20 \%$ below that value. 
Table B.6: Emissions Reduction Sensitivity Reduction $=10 \%$

\begin{tabular}{|l|l|l|l|l|l|l|l|}
\hline$\kappa$ & 0 & 0.2 & 0.4 & 0.6 & 0.8 & 1.0 & 1.2 \\
\hline Cost Ratio & 1.917 & 1.591 & 1.382 & 1.257 & 1.187 & 1.224 & 1.255 \\
\hline$(C / A)^{T P S}$ & 11.97 & 9.94 & 8.63 & 7.85 & 7.41 & 7.64 & 7.83 \\
\hline$\mu$ & 19.60 & 18.32 & 17.11 & 16.09 & 15.09 & 13.98 & 12.91 \\
\hline$I$ & 1099 & 1109 & 1113 & 1111 & 1104 & 1091 & 1075 \\
\hline$E S^{T P S}$ & 191.4 & 191.8 & 192.2 & 192.5 & 192.8 & 193.0 & 193.2 \\
\hline$Q^{T P S}$ & 280.6 & 277.1 & 273.7 & 270.4 & 267.5 & 265.4 & 263.8 \\
\hline$\theta^{T P S}$ & 100.8 & 104.3 & 107.8 & 111.2 & 114.2 & 116.7 & 118.5 \\
\hline Avg $P_{E}^{T P S}$ & 40.89 & 40.31 & 39.85 & 39.46 & 39.13 & 38.89 & 38.71 \\
\hline Tax: $(C / A)^{T a x}=6.24, \tau=12.04, E S=190.0, Q=263.2, \theta=112.9, \mathrm{Avg} P_{E}=45.33$ \\
\hline
\end{tabular}

Reduction $=40 \%$

\begin{tabular}{|l|l|l|l|l|l|l|l|}
\hline$\kappa$ & 0 & 0.2 & 0.4 & 0.6 & 0.8 & 1.0 & 1.2 \\
\hline Cost Ratio & 1.157 & 1.119 & 1.084 & 1.077 & 1.072 & 1.074 & 1.077 \\
\hline$(C / A)^{T P S}$ & 20.86 & 20.17 & 19.54 & 19.41 & 19.33 & 19.36 & 19.42 \\
\hline$\mu$ & 307.28 & 147.83 & 76.90 & 54.20 & 43.05 & 36.94 & 33.16 \\
\hline$I$ & 895 & 855 & 821 & 787 & 756 & 726 & 696 \\
\hline$E S^{T P S}$ & 186.7 & 192.4 & 192.4 & 192.4 & 192.5 & 192.6 & 192.7 \\
\hline$Q^{T P S}$ & 219.0 & 229.3 & 228.5 & 227.9 & 227.3 & 226.4 & 225.1 \\
\hline$\theta^{T P S}$ & 154.6 & 154.5 & 154.1 & 154.5 & 155.1 & 156.3 & 157.9 \\
\hline Avg $P_{E}^{T P S}$ & 58.65 & 42.92 & 43.21 & 43.21 & 43.16 & 43.02 & 42.87 \\
\hline
\end{tabular}

Tax: $(C / A)^{\operatorname{Tax}}=18.03, \tau=37.43, E S=187.2, Q=225.9, \theta=144.7, \operatorname{Avg} P_{E}=56.52$

Baseline : $E S=191.8, Q=284.5, \theta=99.4, \mathrm{Avg} P_{E}=39.96, \mathrm{CO}_{2}=171.3$

Notes: "Tax" and "Baseline" values are for the tax policy and baseline case with no policy, respectively, and are constant across all $\kappa$ values. Remaining values are for the TPS policy. "Cost Ratio" gives the ratio of $(C / A)^{T P S}$ to $(C / A)^{T a x}$. $\mu$ and $\tau$ are in $\$ / t C O_{2}$. ES values are in millions of ES units. $Q$ and $\theta$ values are in million $M W h s$. Avg. $P_{E}$ values give the quantity weighted average electricity prices in $\$ / M W h$. In the "Baseline" row, "COO emissions in million $\mathrm{tCO}_{2}$ and the emissions of the policies are $10 \%$ below that value for the "Reduction $=10 \%$ " case and $40 \%$ below that for the "Reduction $=40 \%$ " case. 